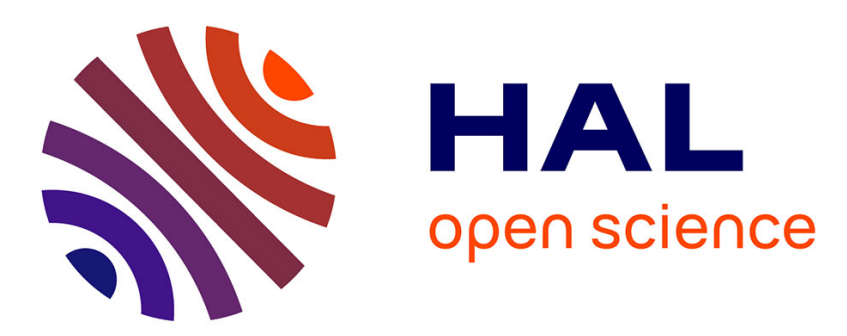

\title{
2D Observer-Based Control of a Vascular Microrobot
}

\author{
Lounis Sadelli, Matthieu Fruchard, Antoine Ferreira
}

\section{To cite this version:}

Lounis Sadelli, Matthieu Fruchard, Antoine Ferreira. 2D Observer-Based Control of a Vascular Microrobot. IEEE Transactions on Automatic Control, 2017, 62 (5), pp.2194-2206. 10.1109/TAC.2016.2604045 . hal-02163251

\section{HAL Id: hal-02163251 \\ https://hal.science/hal-02163251}

Submitted on 24 Jun 2019

HAL is a multi-disciplinary open access archive for the deposit and dissemination of scientific research documents, whether they are published or not. The documents may come from teaching and research institutions in France or abroad, or from public or private research centers.
L'archive ouverte pluridisciplinaire HAL, est destinée au dépôt et à la diffusion de documents scientifiques de niveau recherche, publiés ou non, émanant des établissements d'enseignement et de recherche français ou étrangers, des laboratoires publics ou privés. 


\title{
2D Observer-based Control of a Vascular Microrobot
}

\author{
Lounis Sadelli, Matthieu Fruchard, Antoine Ferreira
}

\begin{abstract}
The paper addresses the $2 \mathrm{D}$ observer-based control of a magnetic microrobot navigating in a cylindrical blood vessel along a reference trajectory. In particular, this robot faces the nonlinear drag force induced by the pulsatile blood flow, which can hardly be measured. Consequently, a mean value theorem (MVT) based observer to estimate the blood velocity from the sole measurement of the robot position is proposed. Also, the stability of the observer-based backstepping controller is proved. The resulting estimation and tracking are then illustrated through simulations, as well as robustness to parametric uncertainty, measurement noise, and dynamical errors when the pulsatile blood flow is incorrectly modeled.
\end{abstract}

Index Terms-observer-based controller, MVT observer, backstepping controller, parametric uncertainty, modeling errors, practical stability, medical robotics

\section{INTRODUCTION}

There has been a growing interest in the development of therapeutic microrobots and nanorobots for some years [1] since such systems can perform complex surgical procedures or diagnosis, reach remote places with lessened medical side effects, and shorten the patient convalescence. Different propulsion strategies have been proposed, mainly based on magnetic deported actuation: elastic flagellum [2], [3], [4], helical flagellum [5], [6], and bead pulled robots or swarm of robots [7], [8].

Whatever the propelling design, such systems face nonlinear forces: electrostatic, contact, and hydrodynamic drag forces [9], [10]. The latter both prevails at a small scale and is the most affected by time-varying perturbations (the pulsatile blood flow). In the synthesis of advanced control laws, the blood velocity is usually assumed to be known or set to a constant mean value, whilst sensitivity studies show that the system is highly sensitive -nonlinearly- to this parameter [9]. A priori knowledge of the blood velocity, either using computational solutions of the Navier-Stokes equations or their analytical approximations is tantalizing. However, the former is unsuitable for real-time control purposes while the latter is very sensitive to the knowledge of the vessel geometry. Another solution is to measure the blood velocity, e.g. using sensors that exploit the Doppler effect [11]. Yet this solution may call for an end-effector servoing to track the robot trajectory. Besides, the sensor spatial and temporal resolution should be high enough to discriminate the blood velocity the robot faces depending on time and on its distance

L. Sadelli and M. Fruchard are with the Laboratory PRISME EA 4229, Univ. Orléans, 63 Av de Lattre de Tassigny, 18020 Bourges Cedex, France, lounis.sadelliduniv-orleans.fr matthieu.fruchardeuniv-orleans.fr

A. Ferreira is with the Laboratory PRISME EA 4229, INSA-CVL, 88 Bd Lahitolle, 18000, Bourges, France. antoine.ferreiradinsa-cvl.fr
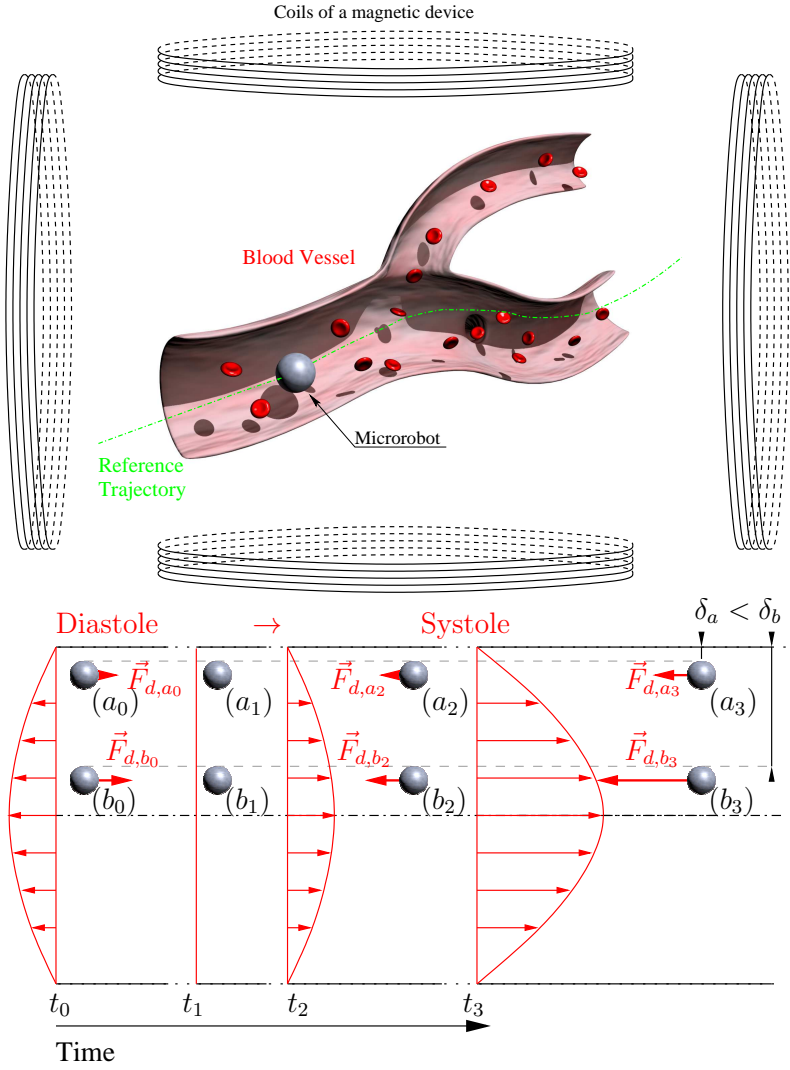

Fig. 1. Pulsatile blood flow: an issue for controlling a magnetic microrobot in a blood vessel along a reference trajectory. Spatial (ordinate) and temporal (abscissa) profiles of the blood flow velocity in a vessel, from the diastolic backflow (minimum negative blood flow velocity) at $t_{0}$ to the systole (maximum blood flow velocity) at $t_{3}$. The figure depicts the drag force acting on stationary microrobots. Spatial discrimination: the microrobots $\left(a_{i}\right)$ and $\left(b_{i}\right)$ do not face the same drag force $\vec{F}_{d}$ because they do not have the same position along the vertical axis $\left(\delta_{a}<\delta_{b}\right)$, except for time $t_{1}$ when $\vec{F}_{d, a_{1}}=\vec{F}_{d, b_{1}}=\overrightarrow{0}$ because the blood velocity is null. Temporal discrimination: the microrobots $\left(b_{0}\right),\left(b_{1}\right),\left(b_{2}\right)$ and $\left(b_{3}\right)$ do not face the same drag force $\vec{F}_{d}$ because the blood flow is pulsatile.

to the vessel walls (see Fig. 1). Conversely, using disturbance rejection approaches is not appropriate since the blood velocity is relevant for control purposes. Considering the blood velocity as an uncertain parameter is an outstanding issue since it is a nonlinearly varying parameter of the drag. In the end, an observer synthesis is appealing to avoid the drawbacks of the aforementioned approaches, if only a blood velocity model is available.

We have previously defined in [12] a dynamic extension of the system in order to model the periodic blood velocity. This extended model has been proved to be observable; we have consequently proposed a receding horizon and a high gain observer to estimate the blood velocity from the sole 
measurement of the robot position, and use it in the control law [12]. Yet, the former lacks from formal convergence proof for this nonlinear system, whilst the latter is known for its output noise sensitivity, especially as the system dimension increases [13]. We have recently proposed an alternative MVT observer-based controller in [14], based on the works of [15], which results in both the stability of the observer-based controller and an improved robustness to output noise. [16] has completed the previous work addressing the robustness to parametric uncertainties.

The present paper generalizes our recent approaches to the 2D system, where more forces are involved, to address the issue of estimating the blood velocity the robot faces depending on its position in the vessel (compare the drag forces on microrobots $a$ and $b$ on Fig. 1). Robustness to uncertain parameters and modeling errors is also investigated. The 2D model of the microrobot is briefly recalled to clarify the problem statement in Section II. Section III is dedicated to the design of an either practically or asymptotically stable MVT observer-based backstepping controller depending on whether the model exhibits or not modeling errors. Simulations results, in Section IV, illustrate the robustness to parametric uncertainty, output noise, disturbance, and modeling errors of the proposed approach. These results are then discussed in Section V.

\section{Modeling and Problem Statement}

We consider a spherical microrobot of radius $r$ and mass $m$, made of ferromagnetic particles and a payload, navigating in a cylindrical blood vessel. The microrobot is actuated using the magnetic motive force $\vec{F}_{m}$ and is subjected to the drag force $\vec{F}_{d}$, the apparent weight $\vec{F}_{w}$, the electrostatic force $\vec{F}_{e}$ and the contact force $\vec{F}_{c}$, depicted on Fig. 2. See e.g. [9] for more details.

We consider a $2 D$ model of the microrobot whose state vector is denoted $x \in \mathbb{R}^{4}$ with $\left[\begin{array}{ll}x_{1} & x_{2}\end{array}\right]^{T}$ the robot position and $\vec{v}=\left[\begin{array}{ll}x_{3} & x_{4}\end{array}\right]^{T}$ the robot velocity in a frame $\mathcal{F}(0, \vec{\imath}, \vec{k})$. The robot translational motion is given by:

$$
m \dot{\vec{v}}=\vec{F}_{d}+\vec{F}_{m}+\vec{F}_{w}+\vec{F}_{e}+\vec{F}_{c} .
$$

Indexes $x$ and $z$ denote forces projections on $\vec{\imath}$ and $\vec{k}$ axis, respectively. Quantities normalized with respect to the robot mass $m$ are followed by a prime symbol, e.g. $F_{d}^{\prime}=\frac{F_{d}}{m}$.

\section{A. Forces}

1) Hydrodynamic force: In the blood, the robot faces the drag force which opposes its motion:

$$
\vec{F}_{d}=-\frac{1}{2} \rho_{f}\left(\frac{\left\|\vec{v}_{r}\right\|^{2}}{\tau_{o}}\right) \frac{\vec{v}_{r}}{\left\|\vec{v}_{r}\right\|} S C_{d}
$$

with $\rho_{f}(\eta)$ the fluid density (viscosity), $S=\pi r^{2}$ the robot frontal area, $\vec{v}_{r}=\vec{v}-\vec{v}_{f}(x, t)$ the relative velocity of the robot with respect to the fluid, and $\tau_{o}$ is a dimensionless ratio related to the partial vessel occlusion by the microrobot, see e.g. [17]. The Reynold's number $R e$ and the drag coefficient $C_{d}$ are given by [18]:

$$
R e=\frac{2 r \rho_{f}\left\|\vec{v}_{r}\right\|}{\tau_{o} \eta} \quad C_{d}=\frac{24}{R e}+\frac{6}{1+\sqrt{R e}}+0.4
$$

Let $\psi=\left(\vec{\imath}, \vec{v}_{r}\right)$ (see Fig. 2); then using (2), we have:

$$
\left\{\begin{array}{l}
\vec{F}_{d}=m\left(F_{d}^{\prime} \cos \psi \vec{\imath}+F_{d}^{\prime} \sin \psi \vec{k}\right)=m\left(F_{d, x}^{\prime} \vec{\imath}+F_{d, z}^{\prime} \vec{k}\right) \\
F_{d}^{\prime}=-\left(a v_{r}+b v_{r}^{2}+c \frac{v_{r}^{2}}{1+d \sqrt{v_{r}}}\right)
\end{array}\right.
$$

with parameters $a, b, c$, and $d$ given by:

$$
a=\frac{9 \eta}{2 \tau_{o} \rho r^{2}} \quad b=\frac{3 \rho_{f}}{20 r \tau_{o}^{2} \rho} \quad c=\frac{9 \rho_{f}}{4 r \tau_{o}^{2} \rho} \quad d=\sqrt{\frac{2 \rho_{f} r}{\tau_{o} \eta}}
$$

Wall effects result in a parabolic flow profile. The pulsatile fluid velocity $v_{f}(x, t)$ is thus modeled as a product of a spatial parabolic shape $v_{s}(x)$ and a time-varying periodic flow $v_{t}(t)$. Arterial pulsatile flow is usually modeled using the Womersley model [19] which results in a truncated Fourier series approximation: any time-varying blood velocity $v_{t}(t)=\xi_{1}$ expressed as an $n$ th-order truncated Fourier series is solution of the autonomous system:

$$
\left(\mathcal{S}_{\xi}\right) \quad\left\{\begin{array}{c}
\dot{\xi}_{2 k-1}=\xi_{2 k}, \quad k=1 \ldots n \\
\dot{\xi}_{2 k}=-\omega^{2}\left(k^{2} \xi_{2 k-1}-k(k+1) \xi_{2 k+1}\right) \\
\vdots \\
\dot{\xi}_{2 n+1}=0
\end{array}\right.
$$

where the mean value is $v_{m}=(n+1) \xi_{2 n+1}$. State $\xi$ remains in a compact set $\mathcal{K}_{\xi} \subset \mathbb{R}^{2 n+1}$. See [12] for details.

2) Magnetic force: Three main propulsion designs for magnetic microrobots have been developped: bead pulled, elastic flagellated, and helical tailed robots, see [1] for a survey. In $2 \mathrm{D}$, their magnetic motive force is given by [9]:

$$
\vec{F}_{m}=\beta_{1} \vec{u}-\beta_{2} \vec{v}_{r}
$$

where $\beta_{2}$ is related to the drag exerting on the helical tail, and is null for elastic flagellated and bead pulled robots. The control input $u \in \mathbb{R}^{2}$ is the magnetic field gradients $\nabla B$ and the frequency of the oscillating magnetic field $B$ for bead pulling and flagellated robots, respectively. $\beta_{1}$ is proportional to the robot magnetization, radius, and ferromagnetic ratio, denoted $\vec{M}, r$, and $\tau_{m}$ respectively. $\beta_{i}$ are positive constants.

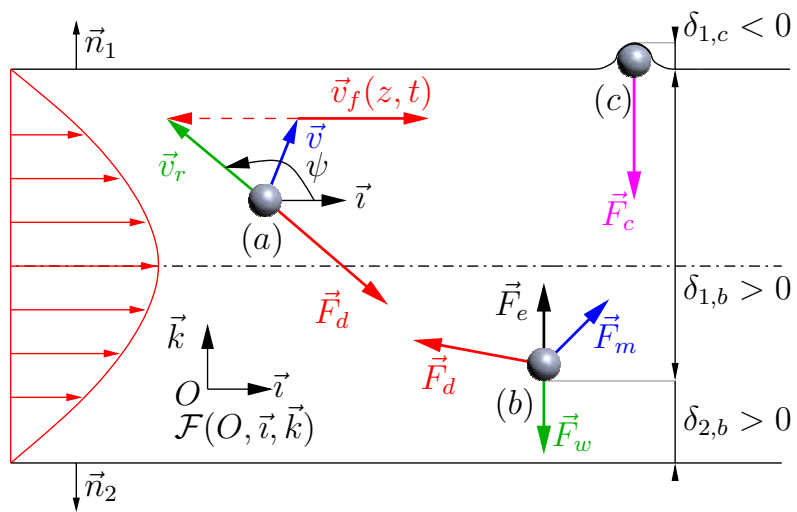

Fig. 2. Forces acting on a microrobot in a $2 D$ cylindrical blood vessel. Algebraic distances from the robot surface to the upper and lower walls are denoted $\delta_{1}$ and $\delta_{2}$ respectively. (a) The relative velocity $\vec{v}_{r}$ of the robot with respect to the fluid is located by the angle $\psi$ in the frame $\mathcal{F}(0, \vec{\imath}, \vec{k})$. The forces acting on the microrobot: hydrodynamic force $\vec{F}_{d}$, magnetic force $\vec{F}_{m}$, apparent weight $\vec{F}_{w}$, electrostatic and contact forces $\vec{F}_{e}$ and $\vec{F}_{c}$, are depicted on (b)-(c). 
3) Apparent weight: The apparent weight of the robot results from the contribution of the weight and the buoyancy:

$$
\vec{F}_{w}=\frac{m}{\rho}\left(\rho-\rho_{f}\right) \vec{g}=-\left(\frac{\rho-\rho_{f}}{\rho}\right) m g \vec{k}=m F_{w}^{\prime} \vec{k}
$$

where the robot density is $\rho=\tau_{m} \rho_{m}+\left(1-\tau_{m}\right) \rho_{p}$ with $\rho_{m}$ and $\rho_{p}$ the magnetic and payload densities, respectively.

4) Electrostatic Force: The electrostatic force between the microrobot and the vessel wall considered as an uncharged surface attracts the robot to the wall:

$$
\vec{F}_{e}=\frac{q^{2}}{4 \pi \varepsilon \varepsilon_{0}}\left[\frac{H(\delta)}{(r+\delta)^{2}}+\frac{H(-\delta)}{r^{2}}\right] \vec{n}
$$

with $H$ the Heaviside step function, $\delta$ the algebraic distance from the robot surface to the wall, $q$ the robot charge, $\varepsilon_{0}$ and $\varepsilon$ are the vacuum and the relative blood's permittivities, respectively. $\vec{n}, \vec{n}_{1}, \vec{n}_{2}$ denote the vector normal to a wall, to the upper and lower wall, respectively (see Fig. 2). The resultant electrostatic interaction with both upper and lower walls, at algebraic distances $\delta_{1}$ and $\delta_{2}$ respectively, is:

$$
\vec{F}_{e}=m e\left(\frac{H\left(\delta_{1}\right)}{\left(r+\delta_{1}\right)^{2}}-\frac{H\left(\delta_{2}\right)}{\left(r+\delta_{2}\right)^{2}}+\frac{H\left(-\delta_{1}\right)-H\left(-\delta_{2}\right)}{r^{2}}\right) \vec{k}
$$

with parameter $e=\frac{3 q^{2}}{16 \pi^{2} r^{3} \rho \varepsilon \varepsilon_{0}}$.

5) Contact force: The contact force is defined by:

$$
\left\{\begin{array}{l}
\vec{F}_{c}=-K|\delta|^{3 / 2} H(-\delta) \vec{n}: \text { loading } \\
\vec{F}_{c}=-F_{\delta_{m}}\left|\frac{\delta}{\delta_{m}}\right|^{3 / 2} H(-\delta) \vec{n}: \text { unloading }
\end{array}\right.
$$

with $F_{\delta_{m}}$ and $\delta_{m}$ the maximum contact force norm and deformation, respectively, and the stiffness $K$. We then have:

$$
\vec{F}_{c}=m f\left(\left|\delta_{2}\right|^{3 / 2} H\left(-\delta_{2}\right)-\left|\delta_{1}\right|^{3 / 2} H\left(-\delta_{1}\right)\right) \vec{k}
$$

with parameter $f=\frac{3 K}{4 \pi r^{3} \rho}$.

\section{B. State representation}

1) Reduced system: The forthcoming reduced system $\left(\mathcal{S}_{r}\right)$ is derived from (1) using forces expressions (3), (5), (6), (7) and (8):

$$
\begin{aligned}
& \left(\mathcal{S}_{r}\right):\left\{\begin{array}{l}
\dot{x}=A_{r} x+B_{r}\left(\beta_{1}^{\prime} u+g(x, \xi)\right)+b_{\vartheta} \vartheta \\
y=C_{r} x
\end{array}\right. \\
& A_{r}=\left[\begin{array}{ll}
0_{2} & I_{2} \\
0_{2} & 0_{2}
\end{array}\right], B_{r}=\left[\begin{array}{c}
0_{2} \\
I_{2}
\end{array}\right], b_{\vartheta}=\left[\begin{array}{c}
0_{31} \\
1
\end{array}\right], C_{r}^{T}=\left[\begin{array}{c}
I_{2} \\
0_{2}
\end{array}\right]
\end{aligned}
$$

where $u=\left[\begin{array}{ll}u_{1} & u_{2}\end{array}\right]^{T}$ is the control input. The output $y=$ $\left[\begin{array}{ll}x_{1} & x_{2}\end{array}\right]^{T}$ is the $2 D$-robot position measured by an imager. The uncertain parameter is here $\vartheta=F_{w}^{\prime}$. The function $g(x, \xi)=$ $\left[\begin{array}{ll}g_{1} & g_{2}\end{array}\right]^{T}$ is given by:

$$
\left\{\begin{array}{l}
g_{1}(x, \xi)=F_{d, x}^{\prime}-\beta_{2}^{\prime}\left(x_{3}-\xi_{1} v_{s}\left(x_{2}\right)\right) \\
g_{2}(x, \xi)=F_{d, z}^{\prime}+F_{e}^{\prime}+F_{c}^{\prime}-\beta_{2}^{\prime} x_{4}
\end{array}\right.
$$

The reduced system $\left(\mathcal{S}_{r}\right)$ models the robot dynamics, and has to be controlled along a reference trajectory.
2) Extended system: Let $\bar{x}$ denote an extended state vector: $\bar{x}=\left[\left[\bar{x}_{1} \ldots \bar{x}_{4}\right]\left[\bar{x}_{4+1} \ldots \bar{x}_{4+2 n+1}\right]\right]=\left[x^{T} \xi^{T}\right]^{T} \in \mathbb{R}^{2 n+5}$. The extended system $\left(\mathcal{S}_{e}\right)$ is inherited from $\left(\mathcal{S}_{r}\right)-\left(\mathcal{S}_{\xi}\right)$, i.e. (9)-(4):

$$
\left(\mathcal{S}_{e}\right):\left\{\begin{aligned}
\dot{\bar{x}} & =A(0) \bar{x}+B\left(\bar{g}(\bar{x})+\beta_{1}^{\prime} u\right)+B_{\vartheta} \vartheta \\
y & =C \bar{x}
\end{aligned}\right.
$$

with $B^{T}=\left[\begin{array}{ll}B_{r}^{T} & 0_{2 \times(2 n+1)}\end{array}\right], B_{\vartheta}^{T}=\left[\begin{array}{ll}b_{\vartheta}^{T} & 0_{1 \times(2 n+1)}\end{array}\right]$, , and $C=\left[\begin{array}{ll}C_{r} & 0_{2 \times 2 n+1}\end{array}\right]$. Function $\bar{g}=\left[\begin{array}{ll}\bar{g}_{1} & \bar{g}_{2}\end{array}\right]^{T}$ is chosen so that $A(0)$ contains the linear part of $\left(\mathcal{S}_{e}\right)$ :

$$
\begin{gathered}
\left\{\begin{array}{l}
\bar{g}_{1}(\bar{x})=g_{1}(\bar{x})+\left(a+\beta_{2}^{\prime}\right)\left(\bar{x}_{3}-\bar{x}_{5}\right) \\
\bar{g}_{2}(\bar{x})=g_{2}(\bar{x})+\left(a+\beta_{2}^{\prime}\right) \bar{x}_{4}
\end{array}\right. \\
A(p)=\left[\begin{array}{ccccccc}
0_{2} & I_{2} & 0_{2} & 0_{2} & \ldots & 0_{2} & 0_{21} \\
A_{1} & A_{2} & A_{3} & 0_{2} & \ldots & 0_{2} & \vdots \\
0_{2} & 0_{2} & \Delta_{1} & \Lambda_{1} & \ddots & \vdots & \vdots \\
\vdots & \vdots & \ddots & \ddots & \ddots & 0_{2} & \vdots \\
\vdots & \vdots & & \ddots & \Delta_{n-1} & \Lambda_{n-1} & 0_{21} \\
0_{2} & 0_{2} & \ldots & \ldots & 0_{2} & \Delta_{n} & A_{4} \\
0_{12} & \ldots & \ldots & \ldots & \ldots & 0_{12} & 0
\end{array}\right] \\
A_{1}=\left[\begin{array}{cc}
0 & p_{1} \\
0 & p_{5}
\end{array}\right], A_{2}=\left[\begin{array}{ccccc}
p_{2} & p_{3} \\
p_{3} & p_{6}
\end{array}\right]-\bar{a} I, A_{3}=\left[\begin{array}{cc}
p_{4}+\bar{a} & 0 \\
p_{7} & 0
\end{array}\right] \\
A_{4}=\left[\begin{array}{cc}
0 \\
n(n+1) \omega^{2}
\end{array}\right], \Delta_{k}=\left[\begin{array}{ccc}
0 & 1 \\
-k^{2} \omega^{2} & 0
\end{array}\right], \Lambda_{k}=\left[\begin{array}{cc}
0 \\
k(k+1) \omega^{2} & 0
\end{array}\right]
\end{gathered}
$$

with $\bar{a}=a+\beta_{2}^{\prime}$ and $p \in \mathbb{R}^{7}$ some parameter whose role will be explained in the next section.

\section{Problem Statement}

To implement a stabilizing control law for the reduced system $\left(\mathcal{S}_{r}\right)$ given by (9) along a given reference trajectory, the state variables $\bar{x}_{1}, \bar{x}_{2}, \bar{x}_{3}, \bar{x}_{4}, \bar{x}_{5}$ are required to be known, that is the robot 2D position and velocity as well as the blood velocity $\bar{x}_{5}=\xi_{1}=v_{t}(t)$. Yet, the former is measured by the imaging device, whereas the latter are not, which justifies the necessity of an observer ${ }^{1}$ of the extended system $\left(\mathcal{S}_{e}\right)$ given by (10). Hence, once a stabilizing controller has been synthesized for system $\left(\mathcal{S}_{r}\right)$, the output feedback problem has to be investigated.

Besides, such a biophysical dependent model is likely to present parametric uncertainties, as illustrated in (9)-(10). Imaging measurement is also affected by output noise. The proposed observer-based controller should address robustness to both of these disturbances.

Finally, the blood velocity is modeled by an $n$ th-order truncated Fourier series as the solution of $\left(\mathcal{S}_{\xi}\right)$ given by (4), yet there is no doubt that the actual periodic blood velocity includes higher harmonic terms. What happens when the blood velocity dynamics are incorrectly modeled?

\footnotetext{
${ }^{1}$ Since the drag force depends nonlinearly on blood velocity, classical assumptions of the adaptive control are broken, and parametric approaches are thus not well-suited for estimating the blood velocity.
} 


\section{MAin RESUlts}

We first state on a technical lemma, which mainly establishes the local controllability of the reduced system (9) and observability of the extended system (10). We then address the main results of the paper: the synthesis of an MVT observer for the extended system $\left(\mathcal{S}_{e}\right)$ with concerns about uncertain parameter, and then the observer-based controller -either asymptotical or practical- semiglobal stability for the reduced system $\left(\mathcal{S}_{r}\right)$, depending on whether the blood velocity dynamics are correctly modeled or affected by modeling errors.

Lemma 1. Let $x_{r e f}=\left[x_{r}(t), \dot{x}_{r}(t), \ddot{x}_{r}(t)\right]$ denote any continuous and bounded reference trajectory, and $\mathcal{K}_{x}$ denote any compact subset of a neighborhood of $\left(x_{r}, \dot{x}_{r}\right)$. Let $\mathcal{U}$ denote the compact set of admissible inputs. $\forall \bar{x} \in \mathcal{K}=\mathcal{K}_{x} \times \mathcal{K}_{\xi}$, $\forall u \in \mathcal{U}$, systems (9) and (10) satisfy the following properties:

P1) The reduced system (9) is locally controllable along $x_{\text {ref }}$

P2) $\bar{g}=\left[\begin{array}{ll}\bar{g}_{1} & \bar{g}_{2}\end{array}\right]^{T}$ is differentiable with respect to $\bar{x}$ and $\forall j \leq 2 n+5, i \leq 2, \exists\left(a_{i, j}, b_{i, j}\right) \in \mathbb{R}^{2}$ such that:

$$
a_{i, j} \leq \frac{\partial \bar{g}_{i}}{\partial \bar{x}_{j}}(\bar{x}) \leq b_{i, j}, a_{i, j} b_{i, j} \leq 0, \forall \bar{x} \in \mathcal{K}
$$

P3) The extended system (10) is such that $(\bar{A}(p), \bar{C})$ is observable $\forall p \in \mathcal{P} \subset \mathbb{R}^{7} \backslash\left\{p_{4}+\bar{a}=0\right\}$ with $\mathcal{P}$ a bounded convex set, and matrices defined as:

$$
\bar{A}(p)=\left[\begin{array}{cc}
A(p) & B_{\vartheta} \\
0_{1 \times(2 n+5)} & 0
\end{array}\right], \quad \bar{C}=\left[\begin{array}{ll}
C & 0_{2 \times 1}
\end{array}\right] .
$$

The proof is given in the Appendix.

Remark 1. Property $\boldsymbol{P} 2$ implies that $\bar{g}$ in (10) is differentiable and locally Lipschitz with respect to the state variable; in turn, the same goes for the function $g$ in (9) (see (11)): $g_{i}$ is locally $\gamma_{i}$-Lipschitz on $\mathcal{K}, i \in\{1,2\}$. The interest of the reformulation of the Lipschitz property in $\boldsymbol{P} 2$ is to provide less conservative conditions in synthesizing the observer gains, as underlined in [15].

Remark 2. Property $\boldsymbol{P} 3$ is related to the local observability, on a bounded convex, of the extended system (10) increased by the parameter dynamical extension.

\section{A. Observer}

In our previous works [20]-[12], we have synthesized high gain observers coupled with adaptive backstepping control laws. Yet, high Lipschitz constants induced by the interaction forces and the high gain matrix formed in ascending powers of the gain for an extended system of higher dimension raise some issues about the resulting sensitivity to noise of these previously proposed observers. Lemma 1 relaxes the conditions for synthesizing the observer (see Remark 1), and we consequently propose the following observer based on [15]. Contrary to our previous approaches, the robustness to parametric uncertainty is not addressed using adaptive control but using state estimation.
Proposition 1 [Observer of the extended system $\left(\mathcal{S}_{e}\right)$ ]. Under assumptions of Lemma $1, \forall \bar{x}(0) \in \mathcal{K}_{0}: \bar{x}(t) \in \mathcal{K} \supset \mathcal{K}_{0}$, $\forall \hat{y}(0) \in \mathcal{K}, \forall \hat{\theta}(0) \in \mathcal{K}_{\vartheta}, \forall u \in \mathcal{U}$,

$$
\left\{\begin{array}{l}
\dot{\hat{y}}=A(0) \hat{y}+B\left(\bar{g}(\hat{\bar{x}})+\beta_{1}^{\prime} u\right)+B_{\vartheta} \hat{\theta}+K_{o}(y-C \hat{y}) \\
\dot{\hat{\theta}}=K_{\theta}(y-C \hat{y}) \\
\hat{\bar{x}}=\operatorname{sat}_{\mathcal{K}}(\hat{y}), \hat{\vartheta}=\operatorname{sat}_{\mathcal{K}_{\vartheta}}(\hat{\theta})
\end{array}\right.
$$

with sat $_{\mathcal{K}}$ a saturation function on the compact $\mathcal{K}$, is an exponential asymptotic observer of $\left(\mathcal{S}_{e}\right)$ on $\mathcal{K} \times \mathcal{K}_{\vartheta}$ if $\exists P_{o}, Q_{o}$ symmetric positive definite and a gain $\bar{K}=\left[\begin{array}{ll}K_{o}^{T} & K_{\theta}^{T}\end{array}\right]^{T}$ that satisfy the Linear Matrix Inequalities (LMIs):

$$
\left(\bar{A}_{i}-\bar{K} \bar{C}\right)^{T} P_{o}+P_{o}\left(\bar{A}_{i}-\bar{K} \bar{C}\right)<-Q_{o}, \forall i \leq 128
$$

with $\bar{A}_{i}=\bar{A}\left(V_{i}\right)$ and $V_{i}$ the vertices of the convex $\mathcal{P}$.

Proof:

Let $\left\{e_{q}(1), \ldots e_{q}(q)\right\}$ denote the canonical basis of $\mathbb{R}^{q}$, and $\epsilon_{1}=\bar{x}-\hat{y}$ denote the estimation error. Let $\operatorname{Co}(\bar{x}, \hat{\bar{x}})=\{\lambda \bar{x}+$ $(1-\lambda) \hat{\bar{x}}, 0 \leq \lambda \leq 1\}$ and $\bar{C} o(\bar{x}, \hat{\bar{x}})$ denote a convex and its convex hull. Applying the mean value theorem to $\bar{g}_{i}, \exists \varrho_{i}(t) \in$ $\bar{C} o(\bar{x}, \hat{\bar{x}})$ :

$$
\bar{g}_{i}(\bar{x})-\bar{g}_{i}(\hat{\bar{x}})=\left(\sum_{j=1}^{2 n+5} e_{2 n+5}^{T}(j) \frac{\partial \bar{g}_{i}}{\partial \bar{x}_{j}}\left(\varrho_{i}(t)\right)\right)(\bar{x}-\hat{\bar{x}})
$$

As $(\bar{x}, \hat{\bar{x}}) \in \mathcal{K}^{2}, \exists \Gamma(t)=\operatorname{diag}\left(\Gamma_{j}(t)\right)$ with $\Gamma_{j}(t) \in(0,1]$ :

$$
\tilde{\bar{x}}=\bar{x}-\hat{\bar{x}}=\Gamma(t) \epsilon_{1}
$$

Since $\bar{g}(\bar{x})=\sum_{i=1}^{2} e_{2}(i) \bar{g}_{i}(\bar{x})$, using (10), (12a)-(12b), (14) and (15), the estimation error satisfies the LPV system:

$$
\left\{\begin{array}{l}
\dot{\epsilon_{1}}=\left(\mathcal{A}(\bar{p}(t))-K_{o} C\right) \epsilon_{1}+B_{\vartheta} \epsilon_{2} \\
\dot{\epsilon_{2}}=-K_{\theta} C \epsilon_{1}
\end{array}\right.
$$

where $\epsilon_{2}=\vartheta-\hat{\theta}$ denotes the parameter estimation error, $\bar{p}_{i, j}(t)=\Gamma_{j}(t) \frac{\partial \bar{g}_{i}}{\partial \bar{x}_{j}}\left(\varrho_{i}(t)\right)$, and the matrix $\mathcal{A}$ is:

$$
\mathcal{A}(\bar{p}(t))=A(0)+B \sum_{i=1}^{2} \sum_{j=1}^{2 n+5} e_{2}(i) e_{2 n+5}^{T}(j) \bar{p}_{i, j}(t)
$$

System (16) can be rewritten as:

$$
\begin{cases}\dot{\epsilon} & =(\overline{\mathcal{A}}(\bar{p}(t))-\bar{K} \bar{C}) \epsilon, \quad \epsilon=\left(\epsilon_{1}^{T} \epsilon_{2}\right)^{T} \\
\overline{\mathcal{A}}(\bar{p}(t)) & =\left[\begin{array}{cc}
\mathcal{A}(\bar{p}(t)) & B_{\vartheta} \\
01 \times(2 n+5) & 0
\end{array}\right]\end{cases}
$$

Property $\mathbf{P} 2$ of Lemma 1 associated with the definition of the $\Gamma_{j}(t)$ implies that $\bar{p}(t)$ evolves in a bounded convex set whose vertices are $\mathcal{V}=\left\{\left(v_{1,1}, \cdots, v_{2,2 n+5}\right): v_{i, j} \in\left\{a_{i, j}, b_{i, j}\right\}\right\}$. Because of the symmetries detailed in the Appendix, this set can be reduced to a convex $\mathcal{P} \subset \mathbb{R}^{7}$ whose $2^{7}$ vertices $V_{i}$ are:

$$
\begin{aligned}
& \mathcal{V}_{\mathcal{P}}=\left\{\quad V=\left(v_{1,2}, v_{1,3}, v_{1,4}, v_{1,5}, v_{2,2}, v_{2,4}, v_{2,5}\right):\right. \\
& \left.v_{i, j} \in\left\{a_{i, j}, b_{i, j}\right\}\right\}
\end{aligned}
$$

For all $P_{o}$ symmetric positive definite, a candidate Lyapunov function (CLF) is given by:

$$
V_{o}(\epsilon)=\epsilon^{T} P_{o} \epsilon \leq \bar{\lambda}\|\epsilon\|^{2}
$$


with $\bar{\lambda}$ the highest eigenvalue of $P_{o}$. Differentiating (18) using (17) leads to: $\dot{V}_{o}(\epsilon)=\epsilon^{T} Q(p(t)) \epsilon$ with the time-varying matrix $Q(p(t))$ affine in $p(t)$ defined by:

$$
Q(p(t))=(\overline{\mathcal{A}}(p(t))-\bar{K} \bar{C})^{T} P_{o}+P_{o}(\overline{\mathcal{A}}(p(t))-\bar{K} \bar{C})
$$

where $\exists \iota_{i} \in[0,1]: \overline{\mathcal{A}}(p(t))=\sum \iota_{i} \bar{A}_{i}$ and $\sum \iota_{i}=1$. From Property $\mathbf{P} 3$ of Lemma 1, $(\bar{A}(p), \bar{C})$ is observable for $p \in \mathcal{P} \subset$ $\mathbb{R}^{7} \backslash\left\{p_{4}+\bar{a}=0\right\}$ : it is a necessary yet not sufficient condition for having the existence of $\bar{K}, P_{o}$ and $Q_{o}$ that satisfy (13). If they exist, we have $Q(V)<-Q_{o} \forall V \in \mathcal{V}_{\mathcal{P}}$. Using the principle of convexity, we then have $Q(p(t))<-Q_{o}, \forall p \in \mathcal{P}$. Hence, we have $\dot{V}_{o}(\epsilon)<-\epsilon^{T} Q_{o} \epsilon$. Let $\underline{\lambda}$ denote the smallest eigenvalue of $Q_{o}$, we then get:

$$
\dot{V}_{o}(\epsilon)<-\underline{\lambda}\|\epsilon\|^{2} \leq-(\underline{\lambda} / \bar{\lambda}) V_{o}(\epsilon)=-k_{o} V_{o}(\epsilon)
$$

It follows that (12) is an exponential observer for system (10).

Remark 3. Property P3, i.e. observability, is only an iff condition for having the existence of matrices $P_{o, i}$ and $Q_{o, i}$ satisfying the $i$-th LMI of (13). Consequently, it is only a necessary yet not sufficient condition for having the existence of unique matrices $P_{o}$ and $Q_{o}$ satisfying all the LMIs.

\section{B. Global stabilizing state feedback}

Had all the $\left(\mathcal{S}_{e}\right)$ states been accessible, we would have synthesized a global asymptotic stabilizing state feedback. We propose a backstepping synthesis [21].

Proposition 2 [State feedback for the reduced system $\left(\mathcal{S}_{r}\right)$ ]. Under assumptions of Lemma 1, the backstepping control law $u_{i}=\kappa_{i}(\bar{x}, \vartheta), i=1,2$ :

$$
\kappa_{i}(\bar{x}, \vartheta)=-\frac{\left(k_{i+2}+k_{i}\right) z_{i+2}+\left(1-k_{i}^{2}\right) z_{i}+g_{i}(\bar{x})+(i-1) \vartheta-\ddot{x}_{i, r}}{\beta_{1}^{\prime}}
$$

with the controller gains $k_{i}, k_{i+2}>0$ and $z_{i}=x_{i}-x_{i, r}$, $z_{i+2}=x_{i+2}+k_{i} z_{i}-\dot{x}_{i, r}$, ensures that the error $z$ exponentially decays to zero for any $\mathcal{C}^{0}$ reference trajectory $x_{r e f}=\left[x_{r}(t), \dot{x}_{r}(t), \ddot{x}_{r}(t)\right]$ and from any bounded initial state $\bar{x}(0)$.

Proof: Let $z_{i}=x_{i}-x_{i, r}, z_{i+2}=x_{i+2}-\dot{x}_{i, r}-\alpha_{i}$ for some stabilizing function $\alpha_{i}$. A first CLF is:

$$
V_{1, i}=\frac{1}{2} z_{i}^{2} \Longrightarrow \dot{V}_{1, i}=z_{i}\left(x_{i+2}-\dot{x}_{i, r}\right)=z_{i}\left(z_{i+2}+\alpha_{i}\right)
$$

Setting $\alpha_{i}=-k_{i} z_{i}$ leads to $\dot{V}_{1, i}=-k_{i} z_{i}^{2}+z_{i} z_{i+2}$. Since $\dot{z}_{i}=x_{i+2}-\dot{x}_{i, r}=z_{i+2}+\alpha_{i}=z_{i+2}-k_{i} z_{i}$, we obtain:

$$
\dot{z}_{i+2}=g_{i}(\bar{x})+(i-1) \vartheta+\beta_{1}^{\prime} u_{i}+k_{i}\left(z_{i+2}-k_{i} z_{i}\right)-\ddot{x}_{i, r}
$$

Then, the second CLF is:

$$
V_{2, i}=V_{1, i}+\frac{1}{2} z_{i+2}^{2}
$$

Differentiating (22) using (21), we obtain:

$$
\begin{aligned}
\dot{V}_{2, i}= & -k_{i} z_{i}^{2}+z_{i+2}\left[g_{i}(\bar{x})+(i-1) \vartheta+\beta_{1}^{\prime} u_{i}\right. \\
& \left.+k_{i} z_{i+2}+\left(1-k_{i}^{2}\right) z_{i}-\ddot{x}_{i, r}\right]
\end{aligned}
$$

Using $u_{i}=\kappa_{i}(\bar{x}, \vartheta)$ given by (20) leads to:

$$
\dot{V}_{2, i}=-k_{i} z_{i}^{2}-k_{i+2} z_{i+2}^{2} \leq-k_{c, i} V_{2, i}
$$

with $k_{c, i}=2 \min \left(k_{i}, k_{i+2}\right)$. Let:

$$
V_{c}=V_{2,1}+V_{2,2}
$$

Using (24), we get:

$$
\dot{V}_{c} \leq-2 \min _{i=1 . .4}\left(k_{i}\right) V_{c}=-k_{c} V_{c}
$$

Hence the asymptotic exponential stability of $z=0$ and thus of the reduced system state $x$ along the reference trajectory.

\section{Semiglobal stabilizing output feedback}

Since some $\left(\mathcal{S}_{e}\right)$ states are not accessible, the state feedback proposed in Proposition 2 is not usable as it is. Thence we address the output feedback semiglobal stability whether or not the blood velocity model $\left(\mathcal{S}_{\xi}\right)$ is affected by modeling errors, see e.g. [21], [22].

Proposition 3 [Asymptotically stabilizing output feedback]. Under assumptions of Proposition 1, the observer-based control law $\hat{u}_{i}=\kappa_{i}(\hat{\bar{x}}, \hat{\vartheta}), i=1,2$ :

$$
\kappa_{i}(\hat{\bar{x}}, \hat{\vartheta})=-\frac{\left(k_{i+2}+k_{i}\right) \hat{z}_{i+2}+\left(1-k_{i}^{2}\right) \hat{z}_{i}+g_{i}(\hat{x})+(i-1) \hat{\vartheta}-\ddot{x}_{i, r}}{\beta_{1}^{\prime}}
$$

ensures the semiglobal exponential asymptotic stability of system (9) along any $\mathcal{C}^{0}$ reference trajectory for any initial state $(\bar{x}(0), \hat{y}(0)) \in \mathcal{K}_{0} \times \mathcal{K}, \hat{\theta}(0) \in \mathcal{K}_{\vartheta}$ with the controller gains $k_{i}, k_{i+2}>0$, and $\hat{z}_{i}=\hat{x}_{i}-x_{i, r}, \hat{z}_{i+2}=\hat{x}_{i+2}+k_{i} \hat{z}_{i}-\dot{x}_{i, r}$.

Proof: Replacing $u_{i}$ with $\hat{u}_{i}$ in (23) and denoting $\tilde{g}_{i}=$ $g_{i}(\bar{x})-g_{i}(\hat{\bar{x}}), \tilde{\vartheta}=\vartheta-\hat{\vartheta}$, we obtain:

$\dot{V}_{2, i}=-k_{i} z_{i}^{2}-k_{i+2} z_{i+2}^{2}+z_{i+2} h_{i+2}(z, \epsilon)$

$h_{i+2}=\tilde{g}_{i}+(i-1) \tilde{\vartheta}+\left(k_{i}+k_{i+2}\right) \tilde{z}_{i+2}+\left(1-k_{i}^{2}\right) \tilde{z}_{i}$

First, let us show that $\forall x(0) \in \mathcal{K}_{x 0}=\left\{x: V_{c}(z) \leq \mu_{c 0}\right\}$, $x(t) \in \mathcal{K}_{x}=\left\{x: V_{c}(z) \leq \mu_{c 0}+\mu_{c}\right\}$. Since $\xi \in \mathcal{K}_{\xi}$ by construction (see (4)), it follows that $\bar{x}(t) \in \mathcal{K}=\mathcal{K}_{x} \times \mathcal{K}_{\xi}$. Let also denote $V_{o}(\epsilon(0)) \leq \mu_{o 0}, \mathcal{K}_{\epsilon}=\left\{\epsilon: V_{o}(\epsilon) \leq \mu_{o 0}+\mu_{o}\right\}$, $\chi=\left[\begin{array}{ll}z^{T} & \epsilon^{T}\end{array}\right]^{T}$ and define the CLF $W_{1}$ based on (18) and (25):

$$
W_{1}(\chi)=\frac{\zeta_{c} V_{c}(z)}{\mu_{c 0}+\mu_{c}-V_{c}(z)}+\frac{\zeta_{o} V_{o}(\epsilon)}{\mu_{o 0}+\mu_{o}-V_{o}(\epsilon)}
$$

Assume that $W_{1} \leq \bar{w}=\zeta_{c} \mu_{c 0} / \mu_{c}+\zeta_{o} \mu_{o 0} / \mu_{o}+1$. Then $W_{1}(z(0), \epsilon(0))<\bar{w}$ and we obtain the induced bounds:

$$
W_{1}(\chi) \leq \bar{w} \Rightarrow V_{c}(z)<\mu_{c 0}+\mu_{c}, V_{o}(\epsilon)<\mu_{o 0}+\mu_{o}
$$

Differentiating (28) leads to:

$$
\dot{W}_{1}=\frac{\zeta_{c}\left(\mu_{c 0}+\mu_{c}\right)}{\left(\mu_{c 0}+\mu_{c}-V_{c}\right)^{2}} \dot{V}_{c}+\frac{\zeta_{o}\left(\mu_{o 0}+\mu_{o}\right)}{\left(\mu_{o 0}+\mu_{o}-V_{o}\right)^{2}} \dot{V}_{o}
$$

with the following bounds on the $\dot{V}_{*}$ factors, where $*$ stands for indexes $c$ or $o$ :

$$
\frac{\zeta_{*}}{\left(\mu_{* 0}+\mu_{*}\right)} \leq \frac{\zeta_{*}\left(\mu_{* 0}+\mu_{*}\right)}{\left(\mu_{* 0}+\mu_{*}-V_{*}\right)^{2}} \leq \frac{\left(\zeta_{*}+\bar{w}\right)^{2}}{\zeta_{*}\left(\mu_{* 0}+\mu_{*}\right)}
$$

Let $h=\left[\begin{array}{llll}0 & 0 & h_{3} & h_{4}\end{array}\right]^{T}$, using (19), (24) and (27), (29) becomes:

$$
\begin{aligned}
& \dot{W}_{1} \leq \Psi(\chi)-\Phi_{c o}(\chi) \\
& \Phi_{c o}(\chi)=\frac{\zeta_{c}}{2\left(\mu_{c 0}+\mu_{c}\right)} k_{c} V_{c}(z)+\frac{\zeta_{o}}{2\left(\mu_{o 0}+\mu_{o}\right)} k_{o} V_{o}(\epsilon) \\
& \Psi(\chi)=-\Phi_{c o}+\left\|\frac{\zeta_{c}\left(\mu_{c 0}+\mu_{c}\right)}{\left(\mu_{c 0}+\mu_{c}-V_{c}\right)^{2}} z^{T}(h(z, \epsilon)-h(z, 0))\right\|
\end{aligned}
$$


Then $\forall \underline{w} \in[0, \bar{w})$, we have $\mathcal{K}_{\chi}=\left\{\chi: \underline{w} \leq W_{1}(\chi) \leq \bar{w}\right\} \subset$ $\mathcal{K}_{x} \times \mathcal{K}_{\epsilon}$. Since $V_{c}$ and $V_{o}$ are positive definite, $\Psi \in \mathcal{C}^{0}$ and its second term is null along $\epsilon=0$ and $z=0$, by continuity we have $\chi \in \mathcal{K}_{\chi} \Rightarrow \Psi<0$ for $k_{o}, k_{c}$ high enough. Hence we have:

$$
\dot{W}_{1} \leq-\Phi_{c o}(\chi), \forall \chi \in \mathcal{K}_{\chi}
$$

Yet, since $\Phi_{c o}$ is positive definite on $\mathcal{K}_{\chi}$, the compact set $\{\chi$ : $\left.W_{1} \leq \bar{w}\right\}$ is a basin of attraction so $\bar{x}(0) \in \mathcal{K}_{0} \Rightarrow \bar{x}(t) \in \mathcal{K}$ and extended state $\chi$ is captured by $\mathcal{K}_{\chi}^{c}=\left\{\chi: W_{1}(\chi) \leq \underline{w}\right\}$. Now that we guarantee that $\bar{x} \in \mathcal{K}$, we can specify the practical stability in $\mathcal{K}_{\chi}^{c}$; since $\forall \bar{x} \in \mathcal{K}, \bar{x}=\operatorname{sat}_{\mathcal{K}}(\bar{x}), \hat{\bar{x}}=\operatorname{sat}_{\mathcal{K}}(\hat{y})$ and $\tilde{g}_{i}$ is a $\mathcal{C}^{1}$ function, using (14) and (15) leads to:

$$
\left\|h_{i+2}(z, \epsilon)\right\|=\varsigma_{i}(\|\epsilon\|), \quad \forall x \in \mathcal{K}_{x}, \forall \epsilon \in \mathbb{R}^{2 n+6}, i \in\{1,2\}
$$

with $\varsigma_{i}$ continuous, bounded, and such that $\varsigma_{i}(0)=0$. Moreover $\exists \nu_{i}: x \in \mathcal{K}_{x} \Rightarrow\left\|z_{i+2}\right\| \leq \nu_{i}$, so we have from (24) and (27):

$$
\dot{V}_{2, i} \leq-k_{c, i} V_{2, i}(z)+\nu_{i} \varsigma_{i}(\|\epsilon\|)
$$

Consider the CLF $W_{2}(z, \epsilon)$ with $\zeta>0$ and $V_{c}$ given by (25):

$$
W_{2}(z, \epsilon)=\zeta V_{c}(z)+V_{o}(\epsilon)
$$

Using (30) and (19), the time derivative of $W_{2}(z, \epsilon)$ satisfies:

$$
\dot{W}_{2}(z, \epsilon) \leq-\zeta k_{c} V_{c}(z)-\left[-\zeta \varsigma(\|\epsilon\|)+\underline{\lambda}\|\epsilon\|^{2}\right]
$$

with $\varsigma(\|\epsilon\|)=\nu_{1} \varsigma_{1}(\|\epsilon\|)+\nu_{2} \varsigma_{2}(\|\epsilon\|)$. Due to the properties of $\varsigma$, there exists $\epsilon_{0}$ such that the bracketed term in (32) is positive definite $\forall \epsilon \geq \epsilon_{0}$. Besides, this $\epsilon_{0}$ can be made arbitrary small through the choice of an arbitrary small ratio $\frac{\zeta}{\lambda}$. Hence we have the semiglobal practical stability of the output feedback.

Lastly, we have to prove the local asymptotic stability. For $\epsilon$ small enough to have $\hat{\bar{x}}=\hat{y} \in \mathcal{K}$ and $\hat{\vartheta}=\hat{\theta}$, using Property $\mathbf{P} 2$ and the Lipschitz constants $\gamma_{i}$ in Remark 1 lead to:

$$
\begin{aligned}
\dot{V}_{2, i} \leq & -k_{i}\left\|z_{i}\right\|^{2}-k_{i+2}\left\|z_{i+2}\right\|^{2} \\
& +\gamma_{i}\left\|z_{i+2}\right\|\|\tilde{\bar{x}}\|+\left(k_{i}+k_{i+2}\right)\left\|z_{i+2}\right\|\left\|\tilde{z}_{i+2}\right\| \\
& +\left(1-k_{i}^{2}\right)\left\|z_{i+2}\right\|\left\|\tilde{z}_{i}\right\|+(i-1)\left\|z_{i+2}\right\|\|\tilde{\vartheta}\|
\end{aligned}
$$

Yet we have the following bound:

$$
\begin{aligned}
z_{i+2}=x_{i+2}+k_{i} z_{i}-\dot{x}_{i, r} & \Longrightarrow \tilde{z}_{i+2}=\tilde{x}_{i+2}+k_{i} \tilde{z}_{i} \\
& \Longrightarrow\left\|\tilde{z}_{i+2}\right\| \leq\left\|\tilde{x}_{i+2}\right\|+k_{i}\left\|\tilde{z}_{i}\right\|
\end{aligned}
$$

So using (34) in (33), bounds (15) and $\|\tilde{\vartheta}\| \leq\left\|\epsilon_{2}\right\|$ yields:

$$
\begin{aligned}
\dot{V}_{2, i} \leq & -k_{i}\left\|z_{i}\right\|^{2}-k_{i+2}\left\|z_{i+2}\right\|^{2} \\
& +\gamma_{i}\left\|z_{i+2}\right\|\|\tilde{\tilde{x}}\|+\left(k_{i}+k_{i+2}\right)\left\|z_{i+2}\right\|\left\|\tilde{x}_{i+2}\right\| \\
& +\left(1+k_{i} k_{i+2}\right)\left\|z_{i+2}\right\|\left\|\tilde{z}_{i}\right\|+(i-1)\left\|z_{i+2}\right\|\|\tilde{\vartheta}\| \\
\leq & -\eta_{i, 1}\|z\|^{2}+\eta_{i, 2}\|z\|\|\epsilon\|
\end{aligned}
$$

with $\eta_{i, 1}=\min \left(k_{i}, k_{i+2}\right)$ and $\eta_{i, 2}=\left(i+\gamma_{i}+k_{i}+k_{i+2}+\right.$ $\left.k_{i} k_{i+2}\right)$. Consider the CLF $W_{2}$ given by (31), using (35) and (19), its time derivative satisfies:

$$
\dot{W}_{2}(z, \epsilon) \leq-\zeta \eta_{1}\|z\|^{2}+\zeta \eta_{2}\|z\|\|\epsilon\|-\underline{\lambda}\|\epsilon\|^{2}
$$

Choosing $\zeta=\eta_{1} \underline{\lambda} / \eta_{2}^{2}$ with $\eta_{1}=\eta_{1,1}+\eta_{2,1} \leq \eta_{2}=\eta_{1,2}+\eta_{2,2}$ thus results in $\dot{W}_{2}(z, \epsilon) \leq-\zeta \eta_{1}\|z\|^{2} / 2-\underline{\lambda}\|\epsilon\|^{2} / 2$. Set $\mu_{1}=$ $\max (\zeta / 2, \bar{\lambda})$ and $\mu_{2}=\min \left(\zeta \eta_{1}, \underline{\lambda}\right) / 2=\frac{\lambda \eta_{1}^{2}}{2 \eta_{2}^{2}}$, we get:

$$
\begin{aligned}
& W_{2}(z, \epsilon) \leq \mu_{1}\left(\|z\|^{2}+\|\epsilon\|^{2}\right) \\
& \dot{W}_{2}(z, \epsilon) \leq-\mu_{2}\left(\|z\|^{2}+\|\epsilon\|^{2}\right)
\end{aligned}
$$

We consequently have $\dot{W}_{2}(z, \epsilon) \leq-\frac{\mu_{2}}{\mu_{1}} W_{2}(z, \epsilon)$. Therefore $z$ and $\epsilon$ exponentially converge to zero, and the same goes for $\tilde{\bar{x}}$ and $\tilde{\vartheta}$ while $x$ converges to $\left(x_{r} \dot{x}_{r}\right)^{T}$; hence (26) ensures the semiglobal asymptotic stability of system (9) along $x_{r e f}$.

Remark 4. It is likely that the actual blood velocity $\xi_{1}^{*}$ will satisfy (4) with $n^{*}$ harmonics, whereas the observer is modeled using (4) with $n<n^{*}$, either to avoid technical issues implied by a high dimensional dynamic extension $\left(\mathcal{S}_{\xi}\right)$, or simply because the unmodeled harmonics amplitudes are some order of magnitude under the first $n$ harmonics amplitudes.

When unmodeled dynamics are considered, the $\left(\mathcal{S}_{\xi}\right)$ odd lines are affected by an unknown bounded harmonic disturbance proportional to $d_{n n^{*}}(t)=\dot{\xi}_{1, n}^{*}$ with high frequency terms only since $\xi_{1, n}^{*}$ denote the actual blood velocity without the first $n$ harmonics and mean value. Note that if $D$ is an upper bound for $\xi_{1, n}^{*}$, then $\left\|d_{n n^{*}}(t)\right\| \leq d_{M}=\omega \frac{n^{*}+n+1}{2} D$. The extended system consequently rewrites as:

$$
\left(\mathcal{S}_{e n n^{*}}\right):\left\{\begin{array}{l}
\dot{\bar{x}}=A(0) \bar{x}+B\left(\bar{g}(\bar{x})+\beta_{1}^{\prime} u\right)+B_{\vartheta} \vartheta+B_{d} d_{n n^{*}}(t) \\
y=C \bar{x}
\end{array}\right.
$$

where $B_{d}$ nonzero entries are only $B_{d, 2 k+5}=\frac{1}{(k+1)}$.

In such a case, asymptotical stability results obtained in previous propositions are relaxed into practical stability.

Proposition 4 [Practically stabilizing output feedback]. Let $d_{M}$ denote an upper bound for $d_{n, n^{*}}(t)$ in (36). Under assumptions of Proposition 1, (12) is an exponential practical observer of system $\left(\mathcal{S}_{\text {enn }}{ }^{*}\right)$ given by (36). Besides, the observer-based control law $\hat{u}_{i}=\kappa_{i}(\hat{\bar{x}}, \hat{\vartheta}), \quad i=1,2$, given by (26) semiglobally practically stabilizes the system (9) in a ball of radius $R_{d}=\sqrt{\frac{2}{3}} \frac{\eta_{2}^{2}}{\eta_{1}^{2}} \frac{\pi \omega\left(n^{*}+n+1\right) D}{k_{0}}$ centered along any $\mathcal{C}^{0}$ reference trajectory $x_{r e f}=\left[x_{r}(t), \dot{x}_{r}(t), \ddot{x}_{r}(t)\right]$.

Proof: Similarly to the proof of Proposition 1, the extended state estimation dynamics satisfy the LPV system:

$$
\dot{\epsilon}=(\overline{\mathcal{A}}(\bar{p}(t))-\bar{K} \bar{C}) \epsilon+\bar{B}_{d} d_{n n^{*}}(t)
$$

with $\bar{B}_{d}=\left[\begin{array}{ll}B_{d}^{T} & 0\end{array}\right]^{T}$. Hence the CLF $V_{o}(\epsilon)$ given by (18) is such that:

$$
\dot{V}_{o}(\epsilon)<-\underline{\lambda}\|\epsilon\|\left(\|\epsilon\|-r_{d}\right)
$$

with $r_{d}=\frac{2 d_{M}}{k_{0}}=\frac{\pi}{\sqrt{6}} \frac{\omega\left(n^{*}+n+1\right) D}{k_{0}}$ since $\left\|B_{d}\right\| \leq \sqrt{\frac{\pi^{2}}{6}}$. It follows from (37) that $\epsilon$ exponentially converges into the ball $B\left(0, r_{d}\right)$. Therefore, (12) is an exponential practical observer for the extended system (36).

Let $W_{1}$ given by (28) denote a CLF for the observer-based controlled system (9) with the control law (26). Whatever $\epsilon$, we obtain boundedness as in the previous proof with an 
additional $\epsilon$-dependent term in $\Psi$, and then using $\bar{\varsigma}(\|\epsilon\|)=$ $-\zeta \varsigma(\|\epsilon\|)-\underline{\lambda} r_{d}\|\epsilon\|$ instead of $\varsigma(\|\epsilon\|)$. Now, let $W_{2}$ given by (31), using (37) and (35) together with the appropriate choice of the constants $\zeta$ and $\mu_{2}$, we obtain:

$$
\begin{aligned}
\dot{W}_{2}(z, \epsilon) & \leq-\zeta \eta_{1}\|z\|^{2} / 2-\underline{\lambda}\|\epsilon\|\left(\frac{\|\epsilon\|}{2}-r_{d}\right) \\
& \leq-\mu_{2}\left(\|z\|^{2}+\|\epsilon\|^{2}\right)+\underline{\lambda} r_{d}\|\epsilon\|
\end{aligned}
$$

Using $\chi=\left[z^{T} \epsilon^{T}\right]^{T}$ and $\mu_{2}=\frac{\lambda}{2 \eta_{1}^{2}}$, (38) gives:

$$
\begin{aligned}
\dot{W}_{2}(z, \epsilon) & \leq-\mu_{2}\|\chi\|^{2}+\underline{\lambda} r_{d}\|\chi\| \\
& \leq-\mu_{2}\|\chi\|\left(\|\chi\|-2 r_{d}\left(\frac{\eta_{2}}{\eta_{1}}\right)^{2}\right)
\end{aligned}
$$

Hence $\chi$ exponentially converges into the ball $B\left(0, R_{d}\right)$ with $R_{d}=\sqrt{\frac{2}{3}} \frac{\eta_{2}^{2}}{\eta_{1}^{2}} \frac{\pi \omega\left(n^{*}+n+1\right) D}{k_{0}}$. Accordingly, $\tilde{\bar{x}}$ and $\tilde{\vartheta}$ exponentially converge into $B\left(0, R_{d}\right)$, and $x$ into $B\left(\left(x_{r} \dot{x}_{r}\right)^{T}, R_{d}\right)$.

TABLE I

NOMINAL PARAMETERS VALUES

\begin{tabular}{|c||c|c|}
\hline Blood viscosity & $\eta$ & $16 \times 10^{-3}[\mathrm{~Pa} . \mathrm{s}]$ \\
\hline Blood density & $\rho_{f}$ & $1060\left[\mathrm{~kg} \cdot \mathrm{m}^{-3}\right]$ \\
\hline Ferromagnetic density & $\rho_{m}$ & $7500\left[\mathrm{~kg} \cdot \mathrm{m}^{-3}\right]$ \\
\hline Robot radius & $r$ & $2.510^{-4}[\mathrm{~m}]$ \\
\hline Vessel diameter & $D$ & $310^{-3}[\mathrm{~m}]$ \\
\hline Payload density & $\rho_{p}$ & $1500\left[\mathrm{~kg} \cdot \mathrm{m}^{-3}\right]$ \\
\hline Ferromagnetic ratio & $\tau_{m}$ & 0.75 \\
\hline Magnetization & $M$ & $1.23 \times 10^{6}\left[\mathrm{A.m} \mathrm{m}^{-1}\right]$ \\
\hline Occlusion ratio & $\tau_{o}$ & 0.65 \\
\hline Stiffness & $K$ & $2.19 \times 10^{3}\left[\mathrm{~Pa} \cdot \mathrm{m}^{\frac{1}{2}}\right]$ \\
\hline Charge & $q$ & $7.13 \times 10^{-11}[\mathrm{C}]$ \\
\hline Blood permittivity & $\varepsilon$ & $70\left[\mathrm{C}^{2} \cdot \mathrm{N}^{-1} \cdot \mathrm{m}^{-2}\right]$ \\
\hline Controller gains & $\left(k_{1}, k_{2}, k_{3}, k_{4}\right)$ & $(7,40,14,20)$ \\
\hline Actuator saturation & $u_{s a t}$ & $0.2\left[\mathrm{~T}^{-1}\right]$ \\
\hline
\end{tabular}

\begin{tabular}{|c|c|c|}
\hline \multirow[b]{2}{*}{$S 1, S 2$} & $\bar{x}_{0}$ & 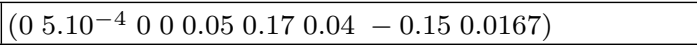 \\
\hline & $\hat{\bar{x}}_{0}$ & 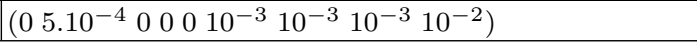 \\
\hline \multirow{2}{*}{$S 2$} & $\vartheta_{0}$ & -8.159 \\
\hline & $\hat{\vartheta}_{0}$ & -7 \\
\hline \multirow{2}{*}{$S 3$} & $\bar{x}_{0}$ & 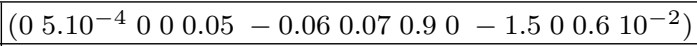 \\
\hline & $\hat{\bar{x}}_{0}$ & $\left(\begin{array}{lllllll}0 & 5.10^{-4} & 0 & 0 & 0 & 10^{-3} & \left.10^{-3} 10^{-3} 10^{-2}\right)\end{array}\right.$ \\
\hline
\end{tabular}

TABLE II

INITIAL CONDITIONS FOR THE SYSTEM AND THE OBSERVER IN Simulation $1(S 1)$, Simulation $2(S 2)$ and Simulation $3(S 3)$.

TABLE III

OUTPUT AND ESTIMATION ERRORS STANDARD DEVIATIONS

\begin{tabular}{|c||c||c||c|}
\hline \multicolumn{1}{|c||}{ Simulation } & $S 1$ & $S 2$ & $S 3$ \\
\hline$y_{1}(\mu m)$ & 100 & 100 & 100 \\
\hline$y_{2}(\mu m)$ & 100 & 50 & 100 \\
\hline$\tilde{\bar{x}}_{1}(\mu m)$ & 44 & 45.44 & 46.26 \\
\hline$\tilde{\bar{x}}_{2}(\mu m)$ & 58.50 & 22.49 & 58.48 \\
\hline$\tilde{\bar{x}}_{3}\left(m m \cdot s^{-1}\right)$ & 1.79 & 6.11 & 4.82 \\
\hline$\tilde{\bar{x}}_{4}\left(m m \cdot s^{-1}\right)$ & 3.96 & 1.36 & 3.37 \\
\hline$\tilde{\bar{x}}_{5}\left(m m \cdot s^{-1}\right)$ & 1.67 & 3.10 & 9 \\
\hline $3 \tilde{\bar{x}}_{9}\left(m m \cdot s^{-1}\right)$ & 0.62 & 3.79 & 3.37 \\
\hline$\tilde{\vartheta}\left(m \cdot s^{-2}\right)$ & - & 0.063 & - \\
\hline
\end{tabular}

\section{Simulation Results}

Without loss of generality, we consider here that the robot is bead pulled, so that we have $\beta_{1}^{\prime}=\frac{\tau_{m} M}{\rho}$ and $\beta_{2}^{\prime}=0$. Nominal parameters values and initial conditions for every simulation are given in Tables I and II, respectively.

In each simulation, a disturbance modeled by an additive acceleration $P_{a}=7 g$ on the $\vec{k}$-axis affects the system in the range $t \in[7,7.001] \mathrm{s}$. For $t \geq 8 \mathrm{~s}$, the output measurements are affected by an additive Gaussian white noise with standard deviations given in Table III so as to model the medical imagers resolution. Simulations are performed by taking into account the actuators limitations. In order to not exceed the magnetic device capacity, the control inputs are time-scaled as $u_{i, a}(t)=u_{i}(t) / k(t)$ with $k(t)=\max _{i=1,2}\left(1,\left|u_{i}(t)\right| / u_{\text {sat }}\right)$. The reference trajectory is defined by:

$$
\left\{\begin{array}{l}
x_{1, r}(t)=0.04 t \\
x_{2, r}(t)= \begin{cases}D / 4 & \text { If } t \leq 5 \\
D / 3 & \text { If } t>5\end{cases}
\end{array}\right.
$$

Remark 5. This trajectory $x_{r e f}=\left[x_{r}(t), \dot{x}_{r}(t), \ddot{x}_{r}(t)\right]$ does not belong to class $\mathcal{C}^{0}$. The interest of a reference trajectory which is close to the upper vessel wall is twofold: first, it minimizes the control efforts (excluding the noise, the control input $u_{2}$ tends to zero on Fig. 3(d) to 3(f) for $t \geq 5$ ); second, output noise is high enough to induce microrobot collisions with the wall to illustrate robustness to strong induced accelerations.

In every single simulation, the blood velocity is modeled by the system $\left(\mathcal{S}_{\xi}\right)$ given by (4) with $n=2$. The first simulation is free of any parametric uncertainty or modeling error and illustrates the robustness to output noise and disturbances. The second one is affected by an additionnal parametric uncertainty, while the last simulation illustrates the effects of modeling errors on the output feedback stability.

\section{A. Simulation 1: robustness to noise output and disturbances}

The MVT observer gain $K_{o}$ given by Proposition 1 is in Table IV. The simulation shown in the first column of Figures 3 and 4 illustrates the results obtained by the MVTobserver when the blood velocity is correctly modeled by a 2nd order truncated Fourier series when there is no parametric uncertainty.

Despite a reference trajectory which is not $\mathcal{C}^{0}$, the observerbased controller is stable since the real and estimated trajectories converge to the reference one after a $2 s$ long transient phase as depicted on Figure 3(a). Despite the $100 \mu m$ standard deviation output noise on the measured position $\left(x_{1}, x_{2}\right)$ and the acceleration disturbance, the real and estimated trajectories are not too much affected and the tracking is efficient. The Figures 4(a) and 4(d) illustrate the reduced system state estimation and tracking errors along the $\vec{i}$ and $\vec{k}$ axes, which converge within $1 s$ and $2 s$, respectively. For time greater than $8 s$, the positions (resp. velocities) estimation errors are respectively less than $165 \mu \mathrm{m}$ and $220 \mu \mathrm{m}$ (resp. $7 \mathrm{~mm} . \mathrm{s}^{-1}$ and $12.5 \mathrm{~mm} . \mathrm{s}^{-1}$ ) along the $\vec{\imath}$ and $\vec{k}$ axes; their standard deviations 
TABLE IV

Observer gain $K_{o}$ in Simulation $1(S 1)$, Simulation $2(S 2)$ and Simulation $3(S 3)$; gain $K_{\theta}$ in $S 2$.

\begin{tabular}{|c|c|c|c|c|c|c|c|c|c|c|c|}
\hline$S 1, S 3$ & $K_{o}^{T}$ & {$\left[\begin{array}{c}3.57 e+2 \\
0\end{array}\right.$} & $\begin{array}{c}0 \\
6.32 e+2\end{array}$ & $\begin{array}{c}2.12 e+4 \\
0\end{array}$ & $\begin{array}{c}0 \\
1.02 e+4\end{array}$ & $\begin{array}{c}1.31 e+4 \\
0\end{array}$ & $\begin{array}{c}1.11 e+5 \\
0\end{array}$ & $\begin{array}{c}2.74 e+3 \\
0\end{array}$ & $\begin{array}{c}-9.63 e+4 \\
0\end{array}$ & $\left.\begin{array}{c}1.68 e+3 \\
0\end{array}\right]$ & \\
\hline$S 2$ & $K_{o}^{T}, K_{\theta}^{T}$ & {$\left[\begin{array}{c}2.81 e+2 \\
0\end{array}\right.$} & $\begin{array}{c}0 \\
3.21 e+2\end{array}$ & $\begin{array}{c}7.02 e+4 \\
0\end{array}$ & $\begin{array}{c}0 \\
1.41 e+4\end{array}$ & $\begin{array}{c}2.50 e+4 \\
0\end{array}$ & $\begin{array}{c}3.39 e+5 \\
0\end{array}$ & $\begin{array}{c}2.75 e+4 \\
0\end{array}$ & $\begin{array}{c}-1.08 e+5 \\
0\end{array}$ & $\left.\begin{array}{c}1.02 e+4 \\
0\end{array}\right]$ & {$\left[\begin{array}{c}0 \\
4.49 e+5\end{array}\right]$} \\
\hline
\end{tabular}

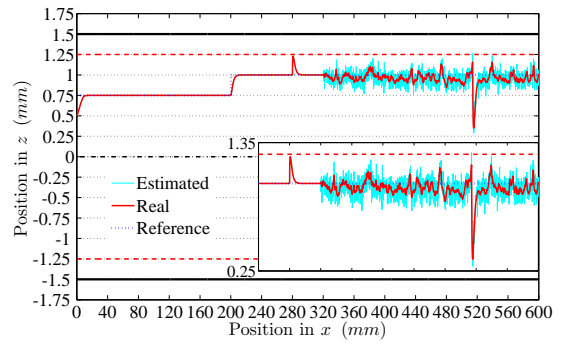

(a) Trajectories

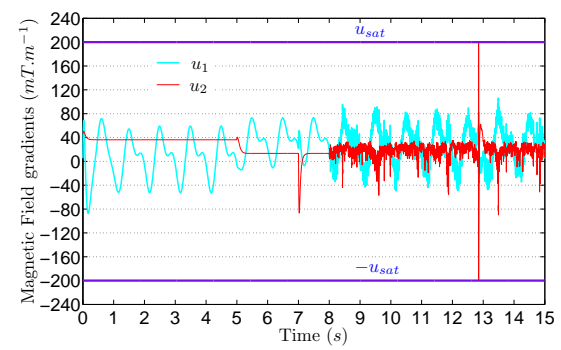

(d) Control input: $\nabla B$.

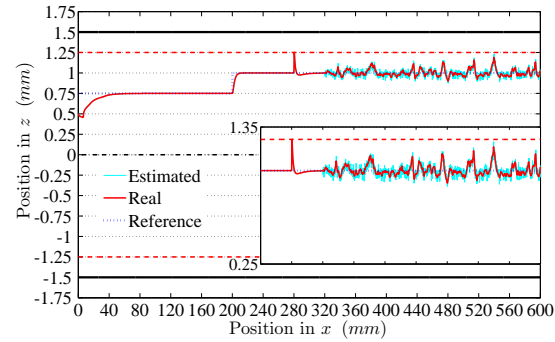

(b) Trajectories

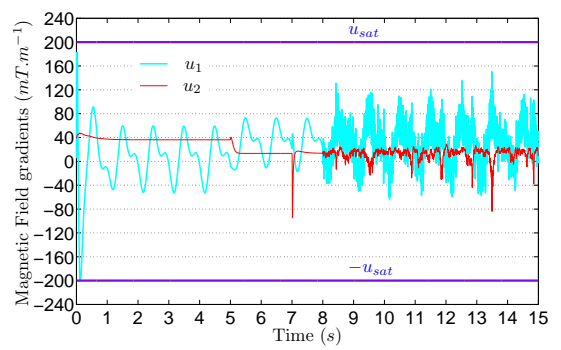

(e) Control input: $\nabla B$.

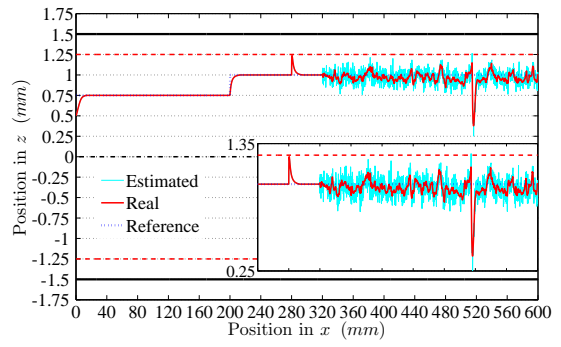

(c) Trajectories

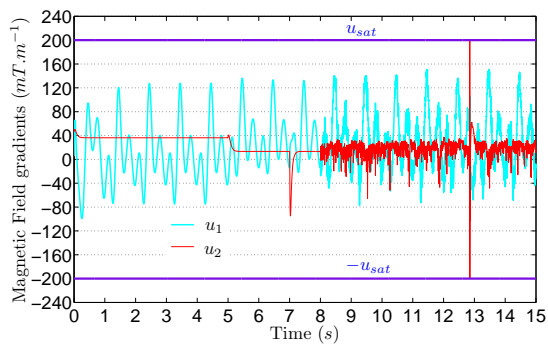

(f) Control input: $\nabla B$.

Fig. 3. Trajectories and control inputs. From left to right: Simulation 1, 2 and 3. From top to bottom: (a)-(c) 2D-trajectory of the microrobot center of gravity, and (d)-(f) the magnetic control inputs $\left(u_{1}, u_{2}\right)$. On subfigures (a) to (c), arterial walls are depicted by thick solid black lines, the red dotted lines depict the limit beyond which the microrobot collides with the walls. The real, estimated and reference trajectories are plotted in thick solid red, thin solid cyan, and blue dash-dotted curves, respectively.

are given in the Table III and show a noticeable improvement with respect to the output noise standard deviation.

The Figure 4(g) illustrates the real and estimated blood velocity $\bar{x}_{5}$ and its mean value $3 \bar{x}_{9}$ and their estimation errors. The estimated blood velocity and its mean value converge to the real ones after $1 s$. When affected by noise, the estimation errors of the blood velocity and its mean value are respectively less than $6 \mathrm{~mm} . \mathrm{s}^{-1}$ and $2.5 \mathrm{~mm} . \mathrm{s}^{-1}$, see Table III for the associated standard deviations. In particular, the $\mathrm{SNR}^{2}$ on the blood velocity (resp. its mean value) is around 30 (resp. 80).

The acceleration disturbance at $t \in[7 ; 7.001] \mathrm{s}$ causes the microrobot to collide the upper wall. Even if the observer does not converge fast enough to estimate correctly this collision, the robot promptly gets back to the reference trajectory. At time $t=12.85 \mathrm{~s}$, the observer estimates that a collision occurs with the upper wall, yet there is none. This dummy collision induces an overestimation of the contact force, and the controller consequently increases the control input $u_{2}$ to counterbalance this force. The control input thus reaches the saturation (see Figure 3(d)), which results in a degraded tracking: the microrobot goes away from the reference trajec-

\footnotetext{
${ }^{2}$ The SNR is here computed as the inverse of the coefficient of variation, i.e. $S N R=\frac{\mu}{\sigma}$ where $\mu$ and $\sigma$ denote the signal mean and its standard deviation, respectively.
}

tory before getting back when the saturation stops. In these two cases, actual or dummy collisions induce peaks in the estimation and tracking errors of the microrobot position and velocity along the $\vec{k}$-axis (see Figures $4(\mathrm{a})-4(\mathrm{~d})$ ).

The control inputs $u_{1}$ and $u_{2}$ are affected by the noise measurement, yet the latter far more than the former as can be noticed in Figure 3(d). The reason for such a difference is that small deviations on the $\vec{k}$-axis position induced by the output noise result in high variations on the estimation of the electrostatic force the robot is very sensitive to, especially when the robot navigates close to the wall (for $t>5 \mathrm{~s}$ ).

\section{B. Simulation 2: Robustness to uncertain parameter}

The MVT observer gains $K_{o}, K_{\theta}$ given by Proposition 1 are in Table IV. This simulation illustrates the results obtained by the MVT-observer when affected by a parametric uncertainty on the apparent weight. Simulation results are depicted on the 2nd column of the Figures 3, 4 and on Figure 5.

The estimated and real trajectories converge to the reference within $2 s$ as shown in Figure 3(b). The position (resp. velocity) estimation and tracking errors along the $\vec{i}$ and $\vec{k}$ axes, depicted on Figure 4(b) (resp.4(e)), are less than $165 \mu \mathrm{m}$ and $80 \mu \mathrm{m}$ (resp. less than $22 \mathrm{~mm} . \mathrm{s}^{-1}$ and $8 \mathrm{~mm} . \mathrm{s}^{-1}$ ); the associated standard deviations are given in Table III. 


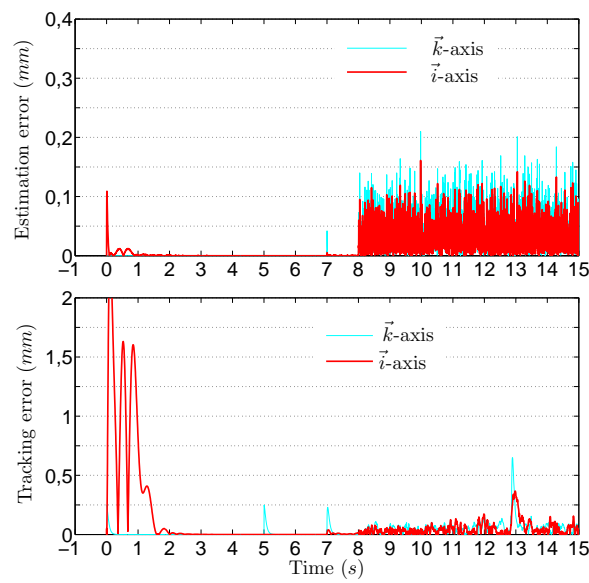

(a) Positions Estimation and tracking errors
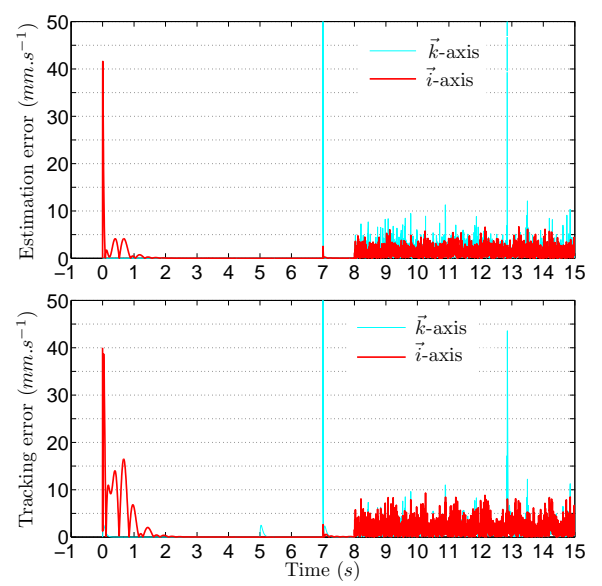

(d) Velocities Estimation and tracking errors

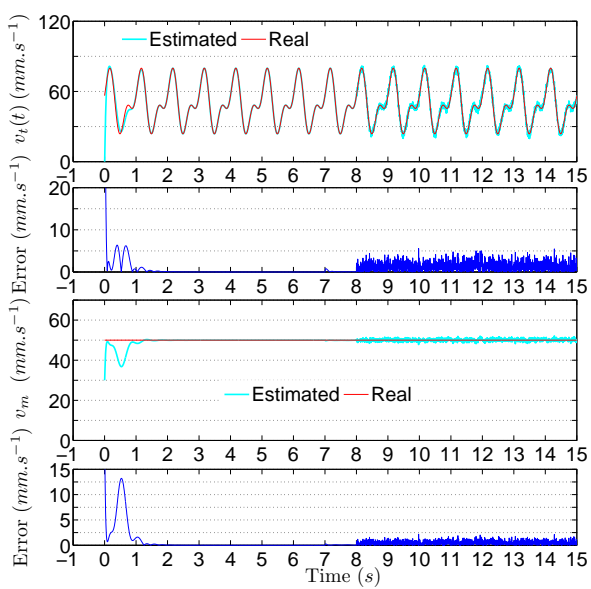

(g) Blood velocity and its mean value

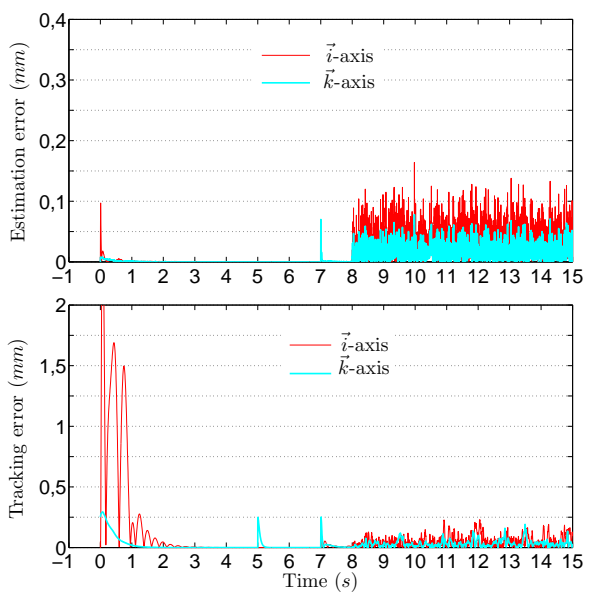

(b) Positions Estimation and tracking errors
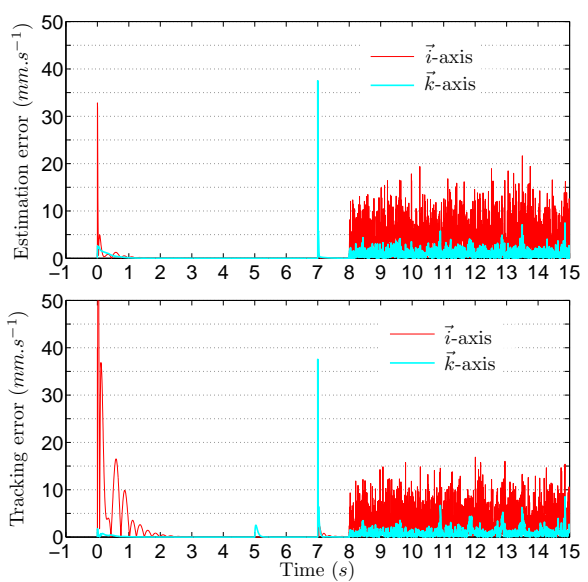

(e) Velocities Estimation and tracking errors

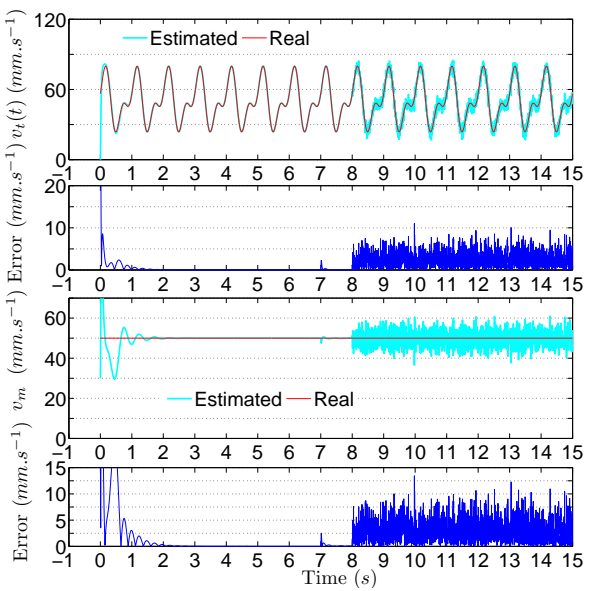

(h) Blood velocity and its mean value

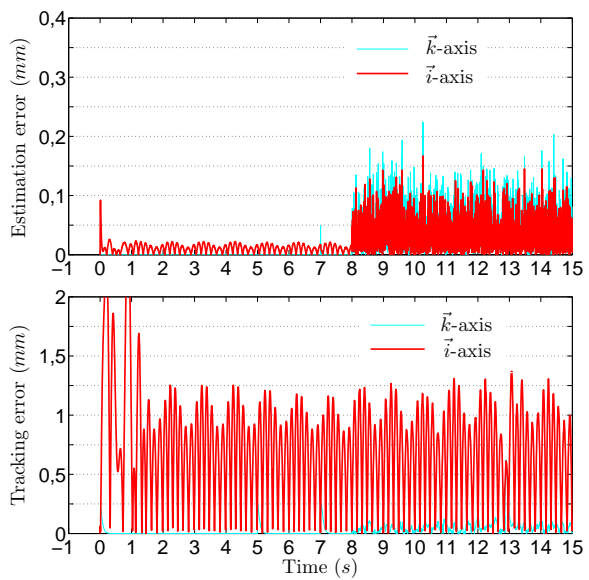

(c) Positions Estimation and tracking errors
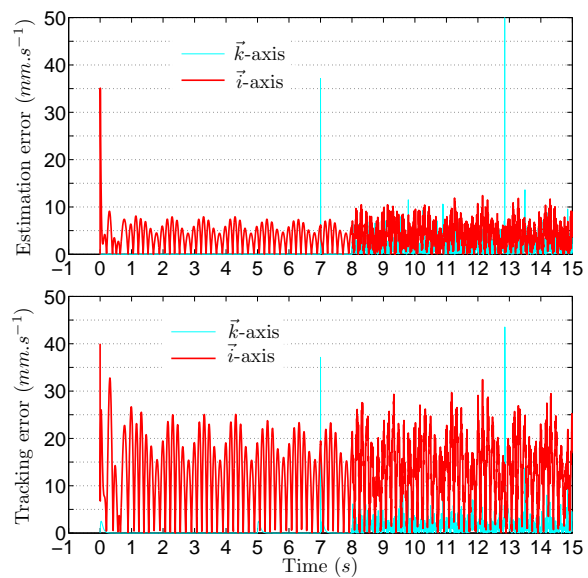

(f) Velocities Estimation and tracking errors

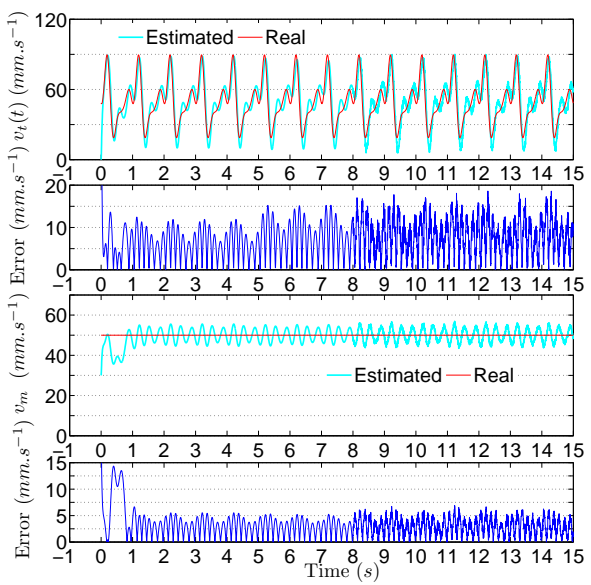

(i) Blood velocity and its mean value

Fig. 4. Errors along $\vec{i}$-axis (thick solid red line) and errors along $\vec{k}$-axis (thin solid cyan line). From left to right: Simulation 1, 2, and 3. From top to bottom: (a)-(c) robot position estimation and tracking errors along the $\vec{\imath}$ and $\vec{k}$ axes, (d)-(f) robot velocity estimation and tracking errors along the $\vec{\imath}$ and $\vec{k}$ axes, (g)-(i) blood velocity and associated estimation error, mean value of the blood velocity and associated estimation error.

The pulsatile blood velocity and its mean value are depicted on Figure 4(h). The estimation errors converge to zero after the transient phase; when affected by output noise, these errors (resp. their SNR) are less than $12 \mathrm{~mm}_{\mathrm{s}} \mathrm{s}^{-1}$ and $14 m m . s^{-1}$ (resp. 16 and 13) -which is degraded compared to the uncertainty-free previous simulation- yet still efficient.

The observer based-controller quickly rejects the acceleration disturbance for $t \in[7,7.001] \mathrm{s}$ : the resulting collision with the upper wall is so quick that it is not estimated by the observer. After this collision, the microrobot separates from 


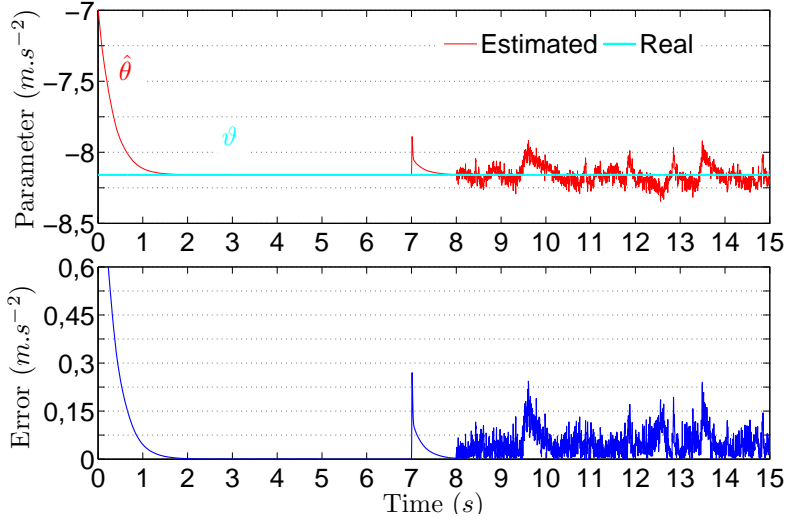

Fig. 5. Simulation 2: (a) Uncertain parameter $\vartheta$, its estimated value, and (b) the associated estimation error.

the wall and gets back to the reference trajectory.

Figure 5 shows the estimation error $\epsilon_{2}=\vartheta-\hat{\theta}$ of the uncertain parameter $\vartheta$. The initial estimation $\hat{\theta}(0)$ is underestimated by one-twelfth with respect to the nominal value, yet converges within $2 s$. This estimation is affected by the disturbance at time $t=7 \mathrm{~s}$, and is very sensitive to the output noise, especially on the $\vec{k}$-axis, because of the high gain $K_{\theta 2}$ (see Table IV) in the update law (12b). The parametric estimation error is less than $0.3 m . s^{-2}$ with a standard deviation given in the Table III and an associated SNR higher than 130.

Both of the control inputs are affected by noise measurement (see Figure 3(e)), and particularly $u_{1}$ because the related gain is higher than in the first simulation. For the second control input, the reason is twofold: the parametric update law (12b) is quite sensitive to noise on the $\vec{k}$-axis, and the reference trajectory is close to the wall for $t>5 \mathrm{~s}$, so that small errors on $x_{2}$ induce big errors on interaction forces estimation. For instance, an underestimation of $x_{2}$ induces an underestimation of the electrostatic force: to counterbalance it, the controller underestimates the control input $u_{2}$, so that the microrobot is even more attracted by the upper wall.

This simulation shows that, despite measurement noise and parametric uncertainty, the MVT-observer provides good estimates and the controller ensures the stability along the reference trajectory.

\section{Simulation 3: Robustness to unmodeled dynamics}

The third simulation is carried out under the same conditions than the first one, except that the actual blood velocity satisfies (4) with $n^{*}=4$, while the observer is still synthesized using (4) with $n=2$. Consequently, the state vectors $x$ and $\hat{x}$ are of dimension $2 n^{*}+5=13$ and $2 n+5=9$, respectively (see Table II). The MVT observer gain is the same as in Simulation 1 (see $K_{o}$ in Table IV). The blood flow velocity is thus incorrectly modeled since the two highest harmonics of the actual signal are not considered in the model: $d_{24}(t) \neq 0$. According to the Remark 4 and Proposition 4, we consequently expect a practical stabilization.

The tracking performances are quite similar to the first simulation ones (compare the first and third columns of Figures 3 and 4), aside from the robot position and velocity along the $\vec{\imath}$-axis which are only practically stabilized because of the modeling errors (see Figures 4(c) and 4(f)). The position (resp. velocity) estimation errors along $\vec{\imath}$-axis and $\vec{k}$-axis are less than $170-225 \mu m$ (resp. $12.5-14 m_{m} . s^{-1}$ ). The SNR are similar than for Simulation 1 (see Table III).

The blood velocity and its mean value, illustrated by the Figure 4(i), converge in less than $1 s$ in a neighborhood of the origin. The associated estimation errors are less than $19 \mathrm{~mm} . \mathrm{s}^{-1}$ and $7 \mathrm{~mm} . \mathrm{s}^{-1}$, with SNR reduced by a factor 6 , yet remaining above 5 and 10 respectively.

The control input $u_{1}$ is not too much affected by noise (see Figure 3(f)) because the robot position and velocity along the $\vec{\imath}$-axis are much more affected by unmodeled dynamics effects than by noise.

\section{DISCUSSION}

All simulations exhibit almost identical trajectory trackings (see Figures 3(a), 3(b) and 3(c)), even if only practical stability is guaranteed for Simulation 3. When disturbances occur, the microrobot collides with the upper wall in every single simulation, yet it is correctly estimated by the observer only in Simulation 1. Despite the modeling errors in Simulation 3 , the tracking is not too much affected as epitomizes Figure 3(c). Even if most of the state estimates are only practically stabilized (i.e. stabilized in a small ball centered at the origin), since the robot position and velocity along the $\vec{k}$-axis are not dependent on the incorrectly modeled blood velocity, the associated estimation errors converge asymptotically as depicted by Figures 4(c) and 4(f).

Let us discuss the choice of the gain matrices $K_{o}$ and $K_{\theta}$. These gains must satisfy the LMIs (13) while trying to have quite low gains -especially on the second column to avoid to inflate noise along the electrostatic force axiswhile keeping reasonable transient phases. In Simulation 2, the same concerns arise additionally for the parametric gain $K_{\theta}$; yet decoupling $K_{o}$ from $K_{\theta}$ is hardly possible. That is why finding the right balance between these antagonist goals induces a transient phase which is twice longer for Simulation 2 , despite a parametric update law highly sensitive to noise because of the high gain $K_{\theta 2}$.

Every single simulation is affected by both output noise and an important disturbance, and illustrates the robustness of the proposed approach with respect to noise and one-shot disturbance. Simulation 2 illustrates the robustness to uncertain parameter, whilst Simulation 3 exemplifies robustness to unmodeled dynamics.

\section{CONCLUSION}

A microrobot immersed in a blood vessel is subjected to various nonlinear forces and above all to the hydrodynamic drag force. Besides the latter nonlinearly depends on the pulsatile blood velocity, which is hardly accessible with accurate enough temporal and spatial resolutions. The navigation of a microrobot in a blood vessel, under the reasonable assumption that its sole position is measured by a medical imager, has been addressed as a trajectory tracking issue for the so-called reduced system, where the control law requires to access some 
unknown states such as the robot velocities and a nonlinearly varying parameter: the pulsatile blood flow velocity.

We have modeled the dynamics of the blood velocity as an $n$ th-order truncated Fourier series with an a priori known pulsation. We have then synthesized an MVT observer for the resulting extended system, thus estimating in particular the required microrobot and blood velocities. The observerbased backstepping control law has been proved to semiglobally stabilize the reduced system along any smooth enough reference trajectory. Since the system depends on biophysical parameters, parametric uncertainties are likely to occur so the proposed observer has been synthesized in order to also estimate a probable unknown parameter. Unless using a high dimensional extended system, the blood velocity model is no doubt truncated with respect to the actual one: we have consequently investigated the effects of such unmodeled dynamics and have obtained a degraded practical stabilization result. The simulation results have illustrated the stability of the proposed observer-based controller and its robustness to output noise, disturbances, parametric uncertainty and modeling errors. The proposed approach can be useful for two purposes: first to perform a surgical task, e.g. the plaques abrasion in clogged arteries, and then for post-operative diagnosis, e.g. to check if the blood velocity is back to normal after the atheroma removal.

Ongoing works include the truncation of the actual blood velocity as well as the additional estimation of the blood pulsation, since it may change over time due to the patient stress. In the latter case, the proposed approach is not well-suited since the resulting model of blood velocity is nonlinear and the associated extended system is no more uniformly observable. Preliminary results [23] using the immersion proposed in [24] are promising, even if its Jacobian invertibility is still to be guaranteed.

\section{APPENDIX}

PROOF OF LEMMA 1

- Property (P1) has been demonstrated in [20]: the local controllability of system (9) is inherited from the controllability of its linearized time-variant system along the reference trajectory $x_{r e f}(t)$ [25], [26].

- Property (P2): In a cylindrical blood vessel, $\psi$ is given by $\psi=\arctan \left[\frac{x_{4}}{x_{3}-v_{f}}\right]$, i.e. $\cos (\psi)=\frac{x_{3}-v_{f}}{v_{r}}$ and $\sin (\psi)=\frac{x_{4}}{v_{r}}$, where $v_{r}=\sqrt{\left[x_{3}-v_{f}\right]^{2}+x_{4}^{2}}$ is the norm of the relative velocity $\vec{v}_{r}$. The blood velocity profile is parabolic along a section of blood vessel of diameter $D$ :

$$
v_{s}\left(x_{2}\right)=1-4\left(\frac{x_{2}}{D}\right)^{2}
$$

Using (3), we define:

$$
\left\{\begin{array}{l}
F_{d 1}^{\prime}=b v_{r}^{2}+c \frac{v_{r}^{2}}{1+d \sqrt{v_{r}}} \\
\frac{\partial F_{d 1}^{\prime}}{\partial v_{r}}=2 b v_{r}+2 c \frac{v_{r}}{1+d \sqrt{v_{r}}}-c d \frac{v_{r}^{3 / 2}}{2\left(1+d \sqrt{v_{r}}\right)^{2}}
\end{array}\right.
$$

We assume that $\forall t \in \mathbb{R}^{+}$, the compact $\mathcal{K}_{x}$ is such that $x_{2} \in\left[-\frac{D}{2}+\varpi, \frac{D}{2}-\varpi\right]$, for some $\left.\varpi \in\right] 0, r[$. We then have $\left(\delta_{1}, \delta_{2}\right) \in[\varpi-r, D-r-\varpi]^{2}$, with $\delta_{1}+\delta_{2}=D-2 r$. To simplify the notation, we will denote:

$$
\begin{array}{ll}
v_{\text {tmax }}=\max _{t}\left(\left|v_{t}\right|\right) & v_{\text {rmax }}=\max _{t}\left(v_{r}\right) \\
x_{2 \max }=\max _{t}\left(\left|x_{2}\right|\right) & \delta_{\text {imax }}=\max _{t}\left(\left|\delta_{i}\right|\right) \\
C_{\psi}=\cos (\psi) & S_{\psi}=\sin (\psi)
\end{array}
$$

Using (39)-(40) we get $0<v_{s}\left(x_{2 \max }\right) \leq v_{s}\left(x_{2}\right) \leq 1$. Using (11), we have the following partial derivatives denoted $\bar{g}_{i, j}=\frac{\partial \bar{g}_{i}}{\partial \bar{x}_{j}}(\bar{x})$ :

$$
\begin{array}{ll}
\bar{g}_{1,1}=\bar{g}_{2,1}=0 & \bar{g}_{1,2}=-\frac{8 x_{2} v_{t}}{D^{2}}\left[\bar{a}+T_{1}\right] \\
\bar{g}_{1,3}=-T_{1} & \bar{g}_{1,4}=\bar{g}_{2,3}=-C_{\psi} S_{\psi} T_{3} \\
\bar{g}_{1,5}=v_{s}\left(x_{2}\right)[T 1+\bar{a}]-\bar{a} & \bar{g}_{2,4}=-T_{2} \\
\bar{g}_{2,2}=\frac{8 x_{2} v_{t}}{D^{2}} C_{\psi} S_{\psi} T_{3}+T_{4} & \bar{g}_{2,5}=v_{s}\left(x_{2}\right) C_{\psi} S_{\psi} T_{3} \\
\bar{g}_{1,2 k+6}=\bar{g}_{2,2 k+6}=0 & \bar{g}_{1,2 k+7}=\bar{g}_{2,2 k+7}=0 \\
\forall k \in\{0, \ldots, n-1\} . &
\end{array}
$$

where the quantities $T_{i}$ are defined by:

$$
\begin{aligned}
& T_{1}=\frac{\partial F_{d 1}^{\prime}}{\partial v_{r}} C_{\psi}^{2}+\frac{F_{d 1}^{\prime}}{v_{r}} S_{\psi}^{2} \\
& =b v_{r}+c \frac{v_{r}}{1+d \sqrt{v_{r}}}+C_{\psi}^{2}\left[b v_{r}+\frac{2 c v_{r}+c d v_{r}^{1.5}}{2\left(1+d \sqrt{v_{r}}\right)^{2}}\right] \\
& T_{2}=\frac{\partial F_{d 1}^{\prime}}{\partial v_{r}} S_{\psi}^{2}+\frac{F_{d 1}^{\prime}}{v_{r}} C_{\psi}^{2} \\
& =b v_{r}+c \frac{v_{r}}{1+d \sqrt{v_{r}}}+S_{\psi}^{2}\left[b v_{r}+\frac{2 c v_{r}+c d v_{r}^{1.5}}{2\left(1+d \sqrt{v_{r}}\right)^{2}}\right] \\
& T_{3}=\frac{\partial F_{d 1}^{\prime}}{\partial v_{r}}-\frac{F_{d 1}^{\prime}}{v_{r}}=b v_{r}+\frac{2 c v_{r}+c d v_{r}^{1.5}}{2\left(1+d \sqrt{v_{r}}\right)^{2}} \\
& T_{4}=2 e\left[\frac{H\left(\delta_{1}\right)}{\left(r+\delta_{1}\right)^{3}}-\frac{H\left(\delta_{2}\right)}{\left(r+\delta_{2}\right)^{3}}\right] \\
& -\frac{3}{2} f\left[\sqrt{\left|\delta_{2}\right|} H\left(-\delta_{2}\right)+\sqrt{\left|\delta_{1}\right|} H\left(-\delta_{1}\right)\right]
\end{aligned}
$$

We consequently have the following bounds on the $T_{i}$ :

$$
\begin{array}{ll}
T_{1} \in\left[0, T_{1}^{+}\right], & T_{1}^{+} \leq 2 b v_{r \max }+2 c v_{r \max }+\frac{c d}{2} v_{r \max }^{1.5} \\
T_{2} \in\left[0, T_{2}^{+}\right], & T_{2}^{+} \leq 2 b v_{r \max }+2 c v_{r \max }+\frac{c d}{2} v_{r \max }^{1.5} \\
T_{3} \in\left[0, T_{3}^{+}\right], & T_{3}^{+} \leq b v_{r \max }+2 c v_{r \max }+\frac{c d}{2} v_{r \max }^{1.5}
\end{array}
$$

To bound $T_{4}$, three cases have to be distinguished:

- if $\left(\delta_{1}, \delta_{2}\right) \in[0 ; D-r-\varpi]^{2}$, we have:

$$
\begin{aligned}
T_{4} & =2 e\left[\frac{1}{\left(r+\delta_{1}\right)^{3}}-\frac{1}{\left(r+\delta_{2}\right)^{3}}\right] \\
& \leq T_{4}^{+}=2 e\left[\frac{1}{r^{3}}-\frac{1}{(D-r)^{3}}\right]
\end{aligned}
$$

- if $\delta_{1} \in[\varpi-r ; 0[$, we have:

$$
\begin{aligned}
T_{4} & =-2 e \frac{1}{\left(r+\delta_{2}\right)^{3}}-\frac{3}{2} f \sqrt{-\delta_{1}} \\
& \geq T_{4,1}^{-}=-2 e \frac{1}{\left(D-r-\delta_{1 \max }\right)^{3}}-\frac{3}{2} f \sqrt{-\delta_{1 \max }}
\end{aligned}
$$

- if $\delta_{2} \in[\varpi-r ; 0[$, we have:

$$
\begin{aligned}
T_{4} & =2 e \frac{1}{\left(r+\delta_{1}\right)^{3}}-\frac{3}{2} f \sqrt{-\delta_{2}} \\
& \geq T_{4,2}^{-}=2 e \frac{1}{\left(D-r-\delta_{2 \max )^{3}}\right.}-\frac{3}{2} f \sqrt{-\delta_{2 \max }}
\end{aligned}
$$

Using (43), (44) and (45), we get bounds on $T_{4}$ :

$$
T_{4} \in\left[T_{4}^{-} ; T_{4}^{+}\right], \quad \text { with } T_{4}^{-}=\min \left(T_{4,1}^{-}, T_{4,2}^{-}\right)
$$

Let $T_{5}^{+}=\left(8 x_{2 \max } v_{\operatorname{tmax}}\right) / D^{2}$. Using the partial derivatives expressions (41) and the bounds (42)-(46), we get 
the bounds $a_{i, j}$ and $b_{i, j}$ on $\frac{\partial \bar{g}_{i}}{\partial \bar{x}_{j}}, \forall k \in\{0, \ldots, n-1\}$ :

$$
\begin{array}{ll}
a_{1,1}=a_{2,1}=0 & b_{1,1}=b_{2,1}=0 \\
a_{1,2}=-T_{5}^{+}\left[\bar{a}+T_{1}^{+}\right] & b_{1,2}=T_{5}^{+}\left[\bar{a}+T_{1}^{+}\right] \\
a_{1,3}=-T_{1}^{+} & b_{1,3}=0 \\
a_{1,4}=a_{23}=-T_{3}^{+} & b_{14}=b_{2,3}=T_{3}^{+} \\
a_{1,5}=\bar{a}\left(v_{s}\left(x_{2 \max }\right)-1\right) & b_{1,5}=T_{1}^{+} \\
a_{2,2}=-\left|T_{5}^{+} T_{3}^{+}+T_{4}^{-}\right| & b_{2,2}=T_{5}^{+} T_{3}^{+}+T_{4}^{+} \\
a_{2,4}=-T_{2}^{+} & b_{2,4}=0 \\
a_{2,5}=-v_{s}\left(x_{2 \max }\right) T_{3}^{+} & b_{2,5}=T_{3}^{+} \\
a_{1,2 k+6}=a_{2,2 k+6}=0 & b_{1,2 k+6}=b_{2,2 k+6}=0 \\
a_{1,2 k+7}=a_{2,2 k+7}=0 & b_{1,2 k+7}=b_{2,2 k+7}=0 .
\end{array}
$$

Besides, using the symmetries and null partial derivatives in (41), it is possible to restrain the study to a lower dimensional vector of parameter $p \in \mathbb{R}^{7}$ defined as:

$$
p=\left[\bar{p}_{1,2}, \bar{p}_{1,3}, \bar{p}_{1,4}, \bar{p}_{1,5}, \bar{p}_{2,2}, \bar{p}_{2,4}, \bar{p}_{2,5}\right]^{T}
$$

- Property (P3): The observability matrix associated with $(\bar{A}(p), \bar{C})$ is given by:

$$
\mathcal{O}=\left[\begin{array}{c|c}
I_{4} & 0_{4 \times(2 n+2)} \\
\hline * & \Theta
\end{array}\right]
$$

where $\Theta \in \mathcal{M}_{(4 n+8) \times(2 n+2)}$, and $*$ stands for some bounded matrice. We then have:

$$
\operatorname{rank}(\mathcal{O})=4+\operatorname{rank}(\Theta)
$$

with

$$
\Theta=\left[\begin{array}{ccccc}
N_{1} & 0_{42} & \ldots & 0_{42} & M_{1} \\
* & N_{2} & \ddots & \vdots & M_{*} \\
\vdots & \ddots & \ddots & 0_{42} & \vdots \\
* & \ldots & * & N_{n} & M_{*} \\
* & \ldots & \ldots & * & Q \\
* & \ldots & \ldots & \ldots & *
\end{array}\right]
$$

and submatrices given by:

$$
\begin{gathered}
N_{k+1}=\varepsilon_{k} \omega^{2 k}\left[\begin{array}{cc}
p_{4}+\bar{a} & 0 \\
p_{7} & 0 \\
* & p_{4}+\bar{a} \\
* & p_{7}
\end{array}\right] M_{1}=\left[\begin{array}{ll}
0 & 0 \\
0 & 1 \\
0 & * \\
0 & *
\end{array}\right] \\
Q=\varepsilon_{n}\left[\begin{array}{cc}
\left(p_{4}+\bar{a}\right) \omega^{2 n} & * \\
p_{7} \omega^{2 n} & * \\
* & * \\
* & *
\end{array}\right] \quad M_{*}=\left[\begin{array}{ll}
0 & * \\
0 & * \\
0 & * \\
0 & *
\end{array}\right]
\end{gathered}
$$

with $\varepsilon_{k}=(k+1) k !^{2}$. Let $D$ denote the matrix composed of the rows $L_{1}, L_{2}, L_{3}, \ldots, L_{2 k+1}, \ldots, L_{4 n+1}$ of $\Theta$. We then have:

$$
|D|=\left[\left(p_{4}+\bar{a}\right)^{2 n+1} \prod_{k=1}^{n-1} \varepsilon_{k}^{2} \omega^{4 k}\right] \varepsilon_{n} \omega^{2 n} .
$$

Hence the sufficient condition on the rank on $\Theta$ :

$$
\omega\left(p_{4}+\bar{a}\right) \neq 0 \Rightarrow \operatorname{rank}(\Theta)=2 n+2
$$

Yet, using the expression of $a_{1,5}$ in (47) and (48), we get:

$$
\bar{g}_{1,5}(t)+\bar{a} \geq v_{s}\left(x_{2 \max }\right)>0 \Rightarrow\left(p_{4}+\bar{a}\right)>0
$$

From (49), (50), and (51), $(\bar{A}(p), \bar{C})$ is observable for any $p \in \mathcal{P} \subset \mathbb{R}^{7} \backslash\left\{p_{4}+\bar{a}=0\right\}$ with $\mathcal{P}$ denoting a convex bounded set.

\section{REFERENCES}

[1] B. J. Nelson, I. K. Kaliakatsos, and J. J. Abbott, "Microrobots for minimally invasive medecine," Annual Review of Biomedical Engineering, vol. 12 , pp. 55-85, 2010.

[2] M. C. Lagomarsino, F. Capuani, and C. P. Lowe, "A simulation study of the dynamics of a driven filament in an aristotelian fluid," Journal of Theoretical Biology, vol. 224(2), pp. 215-224, 2003.

[3] A. A. Evans and E. Lauga, "Propulsion by passive filaments and active flagella near boundaries," Physical Review E, vol. 82(4), p. 041915 , 2010.

[4] R. Dreyfus, J. Beaudry, M. L. Roper, M. Fermigier, H. A. Stone, and J. Bibette, "Microscopic artificial swimmers," Nature, vol. 437, pp. 862 865, 2005.

[5] J. Edd, S. Payen, B. Rubinsky, M. L. Stoller, and M. Sitti, "Biomimetic propulsion for a swimming surgical microrobot," IEEE International Conference on Intelligent Robots and Systems, Las Vegas, pp. 2583$2588,2003$.

[6] L. Zhang, K. E. Peyer, and B. J. Nelson, "Artificial bacterial flagella for micromanipulation," Lab on a chip, vol. 10, no. 17, pp. 2203-2215, 2010.

[7] J. J. Abbott, K. E. Peyer, M. C. Lagomarsino, L. Zhang, L. X. Dong, I. K. Kaliakatsos, and B. J. Nelson, "How should microrobots swim?" International Journal of Robotics Research, vol. 28, pp. 1434-1447, 2009.

[8] J.-B. Mathieu, G. Beaudoin, and S. Martel, "Method of propulsion of a ferromagnetic core in the cardiovascular system through magnetic gradients generated by an mri system," IEEE Transactions on Biomedical Engineering, vol. 53, no. 2, pp. 292-299, 2006.

[9] L. Arcese, M. Fruchard, and A. Ferreira, "Endovascular magneticallyguided robots: navigation modeling and optimization," IEEE Transactions on Biomedical Engineering, vol. 59(4), pp. 977-987, 2012.

[10] P. Vartholomeos and C. Mavroidis, "In silico studies of magnetic microparticle aggregations in fluid environments for mri-guided drug delivery," IEEE Transactions on Biomedical Engineering, vol. 59(11), pp. 3028-3038, 2012.

[11] R. Ponzini, C. Vergara, G. Rizzo, A. Veneziani, A. Roghi, A. Vanzulli, O. Parodi, and A. Redaelli, "Womersley number-based estimates of blood flow rate in doppler analysis: In vivo validation by means of phase-contrast mri," IEEE Transactions on Biomedical Engineering, vol. 57(7), pp. 1807-1815, 2010.

[12] M. Fruchard, L. Arcese, and E. Courtial, "Estimation of the blood velocity for nanorobotics," IEEE Transactions on Robotics, vol. 30, no. 1, pp. 93-102, 2014.

[13] A. Krener and W. Kang, "Locally convergent nonlinear observers," SIAM Journal on Control and Optimization, vol. 42, no. 1, pp. 155-177, 2003.

[14] L. Sadelli, M. Fruchard, and A. Ferreira, "Observer-based controller for microrobot in pulsatile blood flow," IEEE Conference on Decision and Control (CDC 2014), pp. 6993-6998, 2014.

[15] A. Zemouche and M. Boutayeb, "On lmi conditions to design observers for lipschitz nonlinear systems," Automatica, vol. 49, no. 2, pp. 585 591, 2013.

[16] L. Sadelli, M. Fruchard, and A. Ferreira, "Adaptive control of microrobot in pulsatile flow," IEEE International Conference on Control Applications (CCA)- IEEE Multiconference on Systems and Control (MSC 2014), pp. 1982-1987, 2014.

[17] R. Kehlenbeck and R. D. Felice, "Empirical relationships for the terminal settling velocity of spheres in cylindrical columns," Chemical Engineering Technology, vol. 21, pp. 303-308, 1999.

[18] F. White, Viscous Fluid Flow. McGraw Hill New-York, 1991.

[19] J. Womersley, "Method for the calculation of velocity, rate of flow and viscous drag in arteries when the pressure gradient is known," The Journal of physiology, vol. 127(3), pp. 553-563, 1955. 
[20] L. Arcese, M. Fruchard, and A. Ferreira, "Adaptive controller and observer for a magnetic microrobot," IEEE Transactions on Robotics, vol. 29(4), pp. 1060-1067, 2013.

[21] M. Krstić, I. Kanellakopoulos, and P. Kokotović, Nonlinear and Adaptive Control Design. John Wiley \& Sons, Inc., New York, 1995.

[22] A. Teel and L. Praly, "Tools for semiglobal stabilization by partial state and output feedback," SIAM Journal on Control and Optimization, vol. 33(5), pp. 1443-1488, 1995.

[23] L. Sadelli, M. Fruchard, and A. Ferreira, "Estimation de la pulsation cardiaque et la vitesse du sang," in Gèmes Journées Doctorales / Journées Nationales MACS Bourges (France) 2015, 2015.

[24] V. Andrieu, J.-B. Eytard, and L. Praly, "Dynamic extension without inversion for observers," IEEE Conference on Decision and Control, pp. $878-883,2014$.

[25] L. M. Silverman and H. E. Meadows, "Controllability and observability in time-variable linear systems," SIAM Journal on Control and Optimization, vol. 5, pp. 64-73, 1967.

[26] A. Isidori, Nonlinear control systems. Springer-Verlag London, 1995.

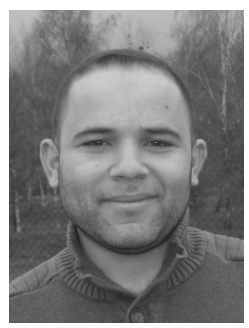

Lounis Sadelli received the Dipl.Ing. and M.S. degrees from the Ecole Nationale Polytechnique of Alger, Alger, Algeria, in 2012, and from the University of Paris XI - Ecole Normale Supérieure de Cachan - Supélec, Paris, France, in 2013, respectively.

$\mathrm{He}$ is currently pursuing the Ph.D. degree with the laboratory PRISME (Laboratoire Pluridisciplinaire de Recherche en Ingénierie des Systèmes, Mécanique et Energétique), University of Orléans, Bourges, France. His research interests are in the modeling and design of nonlinear control laws and observers for healthcare applications.

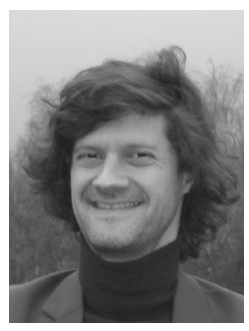

Matthieu Fruchard received the Dipl.Ing. and M.S. degrees from the Ecole Centrale of Lille - University of Lille, France, in 2001, and the Ph.D. degree in control theory from the Ecole des Mines de Paris, Sophia-Antipolis, France, in 2005.

He joined the laboratory PRISME (Laboratoire Pluridisciplinaire de Recherche en Ingénierie des Systèmes, Mécanique et Energétique), University of Orléans, Bourges, France, in 2007, where he is currently Associate Professor. His research interests include control and observer synthesis for classes of nonlinear systems using Lyapunov theory.

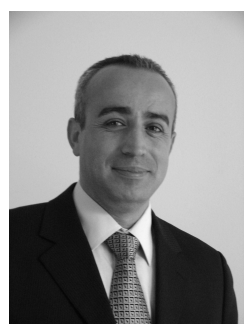

Antoine Ferreira received the M.S. and Ph.D. degrees in electrical and electronics engineering from the University of Franche-Comté, Besancon, France, in 1993 and 1996, respectively. In 1997, he was a Visiting Researcher in the ElectroTechnical Laboratory, Tsukuba, Japan.

$\mathrm{He}$ is currently a Professor of robotics engineering at the laboratory PRISME (Laboratoire Pluridisciplinaire de Recherche en Ingénierie des Systèmes, Mécanique et Energétique), Ecole Nationale Supérieure d'Ingénieurs de Bourges, Bourges, France. He is the author of three books on micro- and nanorobotics and more than 140 journal and conference papers and book contributions. His research interests include the design, modeling, and control of micro- and nanorobotic systems using active materials, micro- and nanomanipulation systems, biological nanosystems, and bionanorobotics. Dr. Ferreira was the Guest Editor for different special issues of the IEEE/ASME TRANSACTIONS ONMECHATRONICS in 2009, International Journal of Robotics Research in 2009, and the IEEE Nanotechnology Magazine in 2008. He is an Associate Editor of Reviews in Advanced Sciences and Engineering. 